University of Nebraska - Lincoln

DigitalCommons@University of Nebraska - Lincoln

2007

\title{
Development of low dissipative high order filter schemes for multiscale Navier-Stokes/MHD systems
}

\author{
Helen C. Yee \\ NASA Ames Research Center, yee@nas.nasa.gov \\ Björn Sjögreen \\ NASA Ames Research Center, Bjorn.Sjogreen@multid.se
}

Follow this and additional works at: http://digitalcommons.unl.edu/nasapub

Yee, Helen C. and Sjögreen, Björn, "Development of low dissipative high order filter schemes for multiscale Navier-Stokes/MHD systems" (2007). NASA Publications. 238.

http://digitalcommons.unl.edu/nasapub/238

This Article is brought to you for free and open access by the National Aeronautics and Space Administration at DigitalCommons@University of Nebraska - Lincoln. It has been accepted for inclusion in NASA Publications by an authorized administrator of DigitalCommons@University of Nebraska - Lincoln. 


\title{
Development of low dissipative high order filter schemes for multiscale Navier-Stokes/MHD systems is
}

\author{
H.C. Yee ${ }^{\mathrm{a}, *}$, B. Sjögreen ${ }^{\mathrm{b}}$ \\ a NASA Ames Research Center, Mail Stop 258-5, Moffett Field, CA, USA \\ ${ }^{\mathrm{b}}$ NADA, Royal Institute of Technology 100 44, Stockholm, Sweden
}

Received 24 August 2006; received in revised form 22 December 2006; accepted 5 January 2007

Available online 27 January 2007

\begin{abstract}
Recent progress in the development of a class of low dissipative high order (fourth-order or higher) filter schemes for multiscale Navier-Stokes, and ideal and non-ideal magnetohydrodynamics (MHD) systems is described. The four main features of this class of schemes are: (a) multiresolution wavelet decomposition of the computed flow data as sensors for adaptive numerical dissipative control, (b) multistep filter to accommodate efficient application of different numerical dissipation models and different spatial high order base schemes, (c) a unique idea in solving the ideal conservative MHD system (a non-strictly hyperbolic conservation law) without having to deal with an incomplete eigensystem set while at the same time ensuring that correct shock speeds and locations are computed, and (d) minimization of the divergence of the magnetic field $(\nabla \cdot \mathbf{B})$ numerical error. By design, the flow sensors, different choice of high order base schemes and numerical dissipation models are stand-alone modules. A whole class of low dissipative high order schemes can be derived at ease, making the resulting computer software very flexible with widely applicable. Performance of multiscale and multiphysics test cases are illustrated with many levels of grid refinement and comparison with commonly used schemes in the literature.
\end{abstract}

(c) 2007 Elsevier Inc. All rights reserved.

Keywords: High order methods; High order filter methods; Multiresolution wavelet analysis of flow data; High-speed shock-turbulence interactions; Richtmyer-Meshkov instability; Navier-Stokes/MHD; Unsteady flows

\section{Introduction}

One of the major stumbling blocks in multiscale shock/turbulence simulations is the lack of proper control of numerical dissipation in existing shock-capturing methods to minimize the smearing of turbulent fluctuations while at the same time coping with strong shocks and steep gradients in a stable and efficient manner. More importantly, existing algorithms and/or new algorithms under development rarely address

\footnotetext{
Expanded version of Proceedings of the Calspace Astronum Conference, March 27-30, 2006, Palm Springs, CA, USA.

* Corresponding author. Tel.: +1 650604 4769; fax: +1 6506044377.

E-mail addresses: yee@nas.nasa.gov (H.C. Yee), bjorns@nada.kth.se (B. Sjögreen).
} 
these issues. Methods commonly used for shock/turbulence interactions relying on switching between spectral or high order compact schemes and shock-capturing schemes are not practical for multiscale shock/turbulence interactions. Frequent switching between these two types of schemes can create severe numerical instability.

For the last decade, the authors and collaborators [53,54,35,41,38,36,55,43,40,56,57,59,61], focused on developing schemes that primarily address overcoming the aforementioned stumbling blocks. Our highly parallelizable class of high order filter schemes does not rely on switching between schemes to avoid the related numerical instability. They have built-in flow sensors in the post processing filter step to control the amount and types of numerical dissipation only where needed, leaving the rest of the flow region free of numerical dissipation. Instead of solely relying on very high order high-resolution shock-capturing methods for accuracy, the filter schemes take advantage of the effectiveness of the nonlinear dissipation contained in good shock-capturing schemes and standard linear filters (and/or high order linear dissipation) as stabilizing mechanisms at locations where needed. The method consists of two steps, a full time step of spatially high order non-dissipative (or very low dissipative) base scheme and an adaptive multistep filter consisting of the products of wavelet based flow sensors and linear and nonlinear numerical dissipations to filter the solution. The adaptive numerical dissipation control idea is very general and can be used in conjunction with spectral, spectral element, finite element, discontinuous Galerkin, finite volume and finite difference spatial base schemes. The type of shock-capturing scheme used as nonlinear dissipation is very general and can be any dissipative portion of high resolution TVD, MUSCL, ENO, or WENO shock-capturing method [52,17,33]. By design, the flow sensors, spatial base schemes and linear and nonlinear dissipation models are stand alone modules. Therefore, a whole class of low dissipative high order schemes can be derived at ease.

There are four subtle attributes of our high order filter approach over standard high order shock-capturing schemes. First, the filter approach utilizes high order conservative discretizations as base schemes with no involvement in flux limiters or Riemann solvers for each full time step discretization. Thus, no knowledge of the eigenstructure of the system is required. For example, we can always solve the conservative MHD system using our base scheme step even though it consists of an incomplete eigensystem set. After the completion of a full time step of the base scheme step, a post-processing filter step is employed. Only the filter step might involve the use of flux limiters and approximate Riemann solvers as stabilizing mechanisms to remove Gibb's phenomena related spurious oscillation resulting from the base scheme step at locations where needed. The flux limiters and approximate Riemann solvers, if needed, are not as crucial as in standard shock-capturing schemes in the sense of ensuring correct shock speeds and shock locations when one is dealing with e.g., the conservative MHD system containing an incomplete set of eigenvectors. This advantage will become more apparent in the next section. Second, the physical viscosity, if present, is automatically taken into consideration by the base scheme step. The amount of filter numerical dissipation will be adjusted accordingly by the flow sensor in the presence of the physical viscosity. Third, the use of a wavelet decomposition of the computed flow data to determine the types of and the location where numerical dissipation is needed is different from most existing numerical schemes in which their numerical dissipation is built into the discretization. In the presence of physical viscosity the more scales that are resolved by the base scheme, the less the filter is utilized, thereby gaining accuracy and computational time. In the limit when all scales are resolved, we are left with a "pure" non-dissipative centered (or very low dissipative) high order spatial scheme. If instead the inviscid part of the equations had been discretized by a scheme with an advanced numerical dissipation model, e.g., the TVD, ENO and WENO schemes, the expensive computation of the numerical dissipation would have been made everywhere in the computational domain, even when dominated by physical viscosity. Fourth, our sixth-order filter procedure in conjunction with the classical fourth-order Runge-Kutta method, in general, requires slightly more CPU time per time step (20\%) than the standard second-order shock-capturing schemes. This is due to the fact that all of our filter schemes require only one Riemann solve per time step per direction (independent of the time discretizations of the base scheme step) as opposed to two Riemann solves per time step per direction by second-order shock-capturing schemes using a second-order Runge-Kutta method. The following gives a general overview of the method with emphasis on our latest development and representative multiscale and multiphysics numerical examples that have not appeared in referred journals. 


\section{Efficient low dissipative high order finite difference solver}

In this section, the scheme for the MHD system in uniform Cartesian grid is summarized. The scheme for gas dynamics is the same except without the three extra magnetic field equations. The high order formulation in generalized moving coordinates with freestream preservation is reported in Vinokur and Yee [49].

\subsection{Solving the conservative system using the symmetrizable eigenvectors}

Consider the 3D conservative and symmetrizable [14,32] (non-conservative) forms of the ideal compressible MHD equations in Cartesian grids,

$$
\begin{array}{ll}
U_{t}+\nabla \cdot \mathbf{F}=0 & \text { (conservative) } \\
U_{t}+\nabla \cdot \mathbf{F}=S & \text { (symmetrizable }), \\
U=\left(\begin{array}{l}
\rho \\
\rho \mathbf{u} \\
e \\
\mathbf{B}
\end{array}\right)_{t} ; \quad \mathbf{F}=\left(\begin{array}{c}
\rho \mathbf{u u}+\left(p+B^{2} / 2\right) I-\mathbf{B B} \\
\mathbf{u}\left(e+p+B^{2} / 2\right)-\mathbf{B B} \cdot \mathbf{u} \\
\mathbf{u B}-\mathbf{B u}
\end{array}\right) ; \quad S=-(\nabla \cdot \mathbf{B})\left(\begin{array}{c}
0 \\
\mathbf{B} \\
\mathbf{B} \cdot \mathbf{u} \\
\mathbf{u}
\end{array}\right) .
\end{array}
$$

Here the velocity vector $\mathbf{u}=(u, v, w)^{\mathrm{T}}$, the magnetic field vector $\mathbf{B}=\left(B_{x}, B_{y}, B_{z}\right)^{\mathrm{T}}, \rho$ is the density, and $e$ is the total energy. The notation $B^{2}=B_{x}^{2}+B_{y}^{2}+B_{z}^{2}$ is used. The superscript " $\mathrm{T}$ " indicates the transpose of the subject row vector.

The pressure is related to the other variables by

$$
p=(\gamma-1)\left(\mathrm{e}-\frac{1}{2} \rho\left(u^{2}+v^{2}+w^{2}\right)-\frac{1}{2}\left(B_{x}^{2}+B_{y}^{2}+B_{z}^{2}\right)\right) .
$$

The magnetic pressure is proportional to $B^{2}$. For plasmas and monatomic gases, $\gamma=5 / 3$. The vector on the right hand side of (2) is the non-conservative portion of the symmetrizable MHD equations and is frequently referred to in the literature as a source term vector.

The conservative and symmetrizable forms of the non-ideal compressible MHD [11] systems (viscous, resistive and Hall MHD) are

$$
\begin{aligned}
& U_{t}+\nabla \cdot \mathbf{F}=\mathbf{F}_{v}, \\
& U_{t}+\nabla \cdot \mathbf{F}=\mathbf{F}_{v}+S, \\
& \mathbf{F}_{v}=\left[\begin{array}{llll}
0 & \operatorname{div} \tau & f_{v 5} & \frac{1}{\sigma}(\triangle \mathbf{B}-\nabla \operatorname{div} \mathbf{B})-\beta_{h} \nabla \times((\nabla \times \mathbf{B}) \times \mathbf{B})
\end{array}\right]^{\mathrm{T}} .
\end{aligned}
$$

The fifth component of $\mathbf{F}_{v}$ is

$$
f_{v 5}=\operatorname{div}\left(\mathbf{u}^{\mathrm{T}} \tau\right)+\operatorname{divh}-\frac{1}{\sigma} \operatorname{div}((\nabla \times \mathbf{B}) \times \mathbf{B})-\beta_{h} \operatorname{div}(((\nabla \times \mathbf{B}) \times \mathbf{B}) \times \mathbf{B}) .
$$

The vector $\mathbf{F}_{v}$ includes viscosity, resistivity, and Hall effect with $\tau$ being the viscous stress tensor, $\sigma$ the conductivity coefficient, $\beta_{h}$ the strength of the Hall effect and $\mathbf{h}$ the heat flux. The plasma $\beta$ is $\beta_{\mathrm{p}}=$ (plasma pressure/magnetic pressure).

Without loss of generality we will describe our numerical methods for the inviscid $x$-flux of the ideal MHD (1) on a uniform grid. The schemes to be discussed, the most part, only spell out the $x$-component terms with the $y$-and $z$-components omitted. Let $A(U)$ denote the Jacobian $\partial F / \partial U$ with the understanding that the present $F$ and $S$ are the inviscid $x$-component of the 3D description above. We also write the non-conservative term $S$ in (2), in the $x$-direction as $N(U) U_{x}$.

An important ingredient in our high order filter method is the use of the dissipative portion of high-resolution shock-capturing schemes as part of the nonlinear filters for accurate capturing discontinuities. If the dissipative portion of higher order Lax-Friedrichs or Nessyahu-Tadmor [28] type of shock-capturing schemes are not employed (see [52] for a discussion), these nonlinear filters usually involve the use of approximate Riemann solvers. 
Seven of the eigenvalues and eigenvectors are identical for the "conservative" Jacobian matrix $A$ and the "symmetrizable" Jacobian matrix $(A-N)$ [12]. For ease of reference, we refer to the distinct eigenvalue (eigenvector) between the conservative and symmetrizable MHD as the eighth eigenvalue (eigenvector). The eighth eigenvector of $A$ of the conservative system associated with the degenerate zero eigenvalue can sometimes coincide with one of the other eigenvectors, thereby making it difficult to obtain a Roe-type approximate Riemann solver for the multi-dimensional conservative MHD. On the other hand, the eigenvectors of the symmetrizable Jacobian $A^{*}=(A-N)$ always form a complete basis, and can be obtained from analytical formulas [14,32] for 1D or higher. Here, a Roe-type average state developed in Gallice [12] for the multi-D symmetrizable MHD is employed to solve both the conservative and symmetrizable systems (1) and (2). This form is an improvement over the Brio and $\mathrm{Wu}$ [2] and Powell [32] forms. See the multistep filter section for more discussion on the rationale of employing symmetrizable eigenvectors to solve the conservative system.

\subsection{Description of high order filter methods}

For non-ideal MHD, we apply the ideal MHD spatial base scheme twice for the viscous flux derivatives (similarly for the resistive and Hall terms). There is no viscous filtering involved. Basically, the filter method consists of two steps, a divergence-free preserving spatial base scheme step (not involving the use of approximate Riemann solvers or flux limiters) and a multistep filter (usually involving the use of approximate Riemann solvers and flux limiters). The high order spatial base scheme to approximate the flux derivative of the ideal MHD is very general. Spectral, spectral element, finite element, discontinuous Galerkin, compact and non-compact schemes are possible candidates. In order to have good shock-capturing capability and improved nonlinear stability related to spurious high frequency oscillations, a multistep filter approach consisting of a high order nonlinear filter and a high order linear filter was investigated in $[55,57,61]$. The nonlinear filter consists of the product of an artificial compression method (ACM) indicator or wavelet (WAV) sensor [38] and the nonlinear dissipative portion of a high-resolution shock-capturing scheme. The high order linear filter consists of the product of another sensor and a spectral-like filter or a high order centered linear dissipative operator that is compatible with the order of the base scheme being used.

\subsubsection{Divergence-free preserving base scheme step}

The first step of the numerical method consists of a time step via a high order non-dissipative spatial and high order temporal base scheme operator $L^{*}$. After the completion of a full time step of the base scheme step, the solution is denoted by $U^{*}$

$$
U^{*}=L^{*}\left(U^{n}\right),
$$

where $U^{n}$ is the numerical solution vector at time level $n$. For strong shock interactions and/or steep gradient flows, a small amount of high order linear dissipation can be added to the base scheme step to reduce the time step constraint and stability. For example, an eighth-order linear dissipation with the sixth-order centered non-compact and compact base schemes to approximate $F(U)_{x}$ (with the grid indices $k$ and $l$ for the $y$-and $z$-directions suppressed) is written as

$$
\begin{aligned}
& \frac{\partial F}{\partial x} \approx D_{06} F_{j}+d(\Delta x)^{7}\left(D_{+} D_{-}\right)^{4} U_{j}, \\
& \frac{\partial F}{\partial x} \approx C_{06} F_{j}+d(\Delta x)^{7}\left(D_{+} D_{-}\right)^{4} U_{j},
\end{aligned}
$$

where $D_{06}$ is the standard sixth-order accurate centered difference operator, and $D_{+} D_{-}$is the standard secondorder accurate centered approximation of the second derivative. The small parameter $d$ is a scaled value (e.g., spectral radius of $A(U)$ ) in the range of $0.00001-0.0005$, depending on the flow problem, and has the sign which gives dissipation in the forward time direction. The $D_{06}$ operator is modified at boundaries in a stable way by the so called summation-by-part (SBP) operators [30,29,55]. The linear numerical dissipation operator $D_{+} D_{-}$is modified at the boundaries to be semi-bounded [36]. This highly accurate spatial base scheme is employed to numerically preserve the divergence-free condition of the magnetic field (to the level of round-off error) for uniform Cartesian grids with periodic boundary conditions. The symbol $C_{06}$ in (6) denotes the sixth-order centered compact operator. Comparison of the two base schemes are reported in [61]. 


\subsubsection{Multistep linear and nonlinear filters (suppression of high frequency oscillations and shock-capturing)}

For generality of discussion, we denote the standard spectral filter, compact filter and non-compact high order linear numerical dissipation as high order linear numerical dissipations (or linear filter). In contrast, we denote the dissipative portion of any high resolution shock-capturing scheme as nonlinear numerical dissipation since these dissipations are nonlinear even if one applies the scheme to a linear conservation law. When nonlinear dissipations are applied in a filter approach (to be discussed), we denote the approach as nonlinear filters. Although nonlinear numerical dissipations can suppress spurious high frequency oscillations, they might not be as effective as the standard high order linear dissipations (or linear filters). With the appropriate wavelet flow sensors, they can detect locations of spurious high frequency oscillations, locations of shocks and high gradient regions, and locations of large vortices or vortex sheets separately. The appropriate numerical dissipations are then applied to these locations with the remaining regions free of numerical dissipation. See $[38,55,57]$ for a discussion.

Blending of different types of numerical dissipations - single step linear and nonlinear filter: In the early stages of our development, we proposed the blending of these two types of numerical dissipation into a single filter step after a complete full time step of the base scheme step (or after each stage of the temporal discretization if multistage temporal discretizations were employed), see [55,57] and references cited therein. Subsequent studies [57,61] showed that the blending of more than one type of filter in a single step might create numerical instability due to the frequent switching of filters. For the MHD system, the single step blending of more than one filter can interfere with the divergence-free preserving property as discussed above.

Multistep filters: If instead, we apply the linear filter and nonlinear filter in a separate step, numerical stability is greatly improved. Moreover, the interfering of the divergence-free property is minimized. Our recent study indicates that a multistep filter, e.g., applying the nonlinear filter step after the high order linear filter step in sequence (or vice versa) is more effective than the blending of different types of numerical dissipation in a single step. Studies in Yee and Sjögreen [61] indicated that if the compact base scheme (6) were used for complex shock interactions, the multistep filter is needed (a linear compact filter step and a nonlinear filter step).

The multistep filter or the single step filter can be applied (a) after each stage of a multistage temporal discretization (if such time discretization will be used), or (b) after the completion of each full time step of the mulstistage time discretization. Both options were implemented and tested on a wide variety of gas dynamics and MHD problems. Studies indicated that even if multistage Runge-Kutta methods are employed, there is no advantage in employing the filter step "after each Runge-Kutta stage" over the application of the filter step "after a full time step" of the Runge-Kutta method. On the other hand, option (b) is extremely efficient since only one Riemann solve per time step per dimension is required. The next section discusses filter option (b) with filter option (a) similarly. In addition, the following only gives a description of the nonlinear filter step. It is understood that if the high order linear filter step is employed, the procedure follows the same vein. See [57] for the formula. Before the description of the adaptive nonlinear filter step, we would like to discuss our procedure for solving the conservative system and the symmetrizable system.

Solving the conservative system using the symmetrizable eigenvectors

This class of filter schemes is suitable for solving both conservative and symmetrizable non-conservative systems. In solving the symmetrizable system, the base scheme and the filter step are applied to the non-conservative system (2) with a complete set of eigenvectors. However, for strong shocks, to ensure the correct shock strength and location, we prefer to solve the conservative MHD system. In solving the conservative system, the base scheme step presents no problem. The question is how to overcome the incomplete set of eigensystem issue if nonlinear filters involving Riemann solvers are required? In this case, as described in $[40,57,56]$, we use eigenvectors of the symmetrizable form but with the degenerate eigenvalue replaced by an entropy correction (a small parameter $\epsilon$ that is scaled by the largest eigenvalue of $A(U)$ ) for the conservative form. For more than one space dimension, a multi-dimensional entropy correction [52] is used for each of the degenerate eigenvalues in each spatial direction. Our rationale for doing this is that both systems share the same eigenvalues and eigenvectors except one. The incorrect eigenvector for the conservative form will be multiplied by an eigenvalue which is close to zero. Thus the effect of this "false" eigenvector will be small. (Note that in the present context, the use of an entropy correction is different from the standard entropy correction associated with expansion shocks in the Roe-type approximate solver in gas dynamics.) Another rationale is that solving 
the conservative system by the base scheme step has already ensured the correct shock speed and location of the solution. In turn, the flow sensor is sensing the resulting solution with the correct locations where shockcapturing dissipation is needed. The use of shock-capturing dissipation here is a post-processing step. It plays a different role than if one solves the conservative system by its full shock-capturing scheme counterpart (using the same false eigenvector).

\subsubsection{Adaptive nonlinear filter step (discontinuities and high gradient capturings)}

After the completion of a full time step of the divergence-free preserving base scheme step, the second step is to adaptively filter the solution by the product of a "wavelet sensor" and the "nonlinear dissipative portion of a high-resolution shock-capturing scheme" (involving the use of flux limiters). The final update step after, e.g., the nonlinear filter step can be written (with some grid indices suppressed for ease of illustration) as

$$
U_{j, k, l}^{n+1}=U_{j, k, l}^{*}-\frac{\Delta t}{\Delta x}\left[H_{j+1 / 2}^{f x}-H_{j-1 / 2}^{f x}\right]-\frac{\Delta t}{\Delta y}\left[H_{k+1 / 2}^{f y}-H_{k-1 / 2}^{f y}\right]-\frac{\Delta t}{\Delta z}\left[H_{l+1 / 2}^{f z}-H_{l-1 / 2}^{f z}\right] .
$$

Here, $H_{j \pm 1 / 2}^{f x}, H_{k \pm 1 / 2}^{f y}$ and $H_{l \pm 1 / 2}^{f z}$ are the filter numerical fluxes in the $x, y$ and $z$-directions, respectively. The $x$ filter numerical flux vector $H_{j+1 / 2}^{f x}$ is

$$
H_{j+1 / 2}^{f x}=R_{j+1 / 2} \bar{H}_{j+1 / 2},
$$

where $R_{j+1 / 2}$ is the matrix of right eigenvectors of the Jacobian of the non-conservative MHD flux vector $\left(A_{j+1 / 2}-N_{j+1 / 2}\right)$ evaluated at the Gallice average state [12] in terms of the $U^{*}$ solution from the base scheme step (4). The subscript in $R_{j+1 / 2}$ indicates the average state evaluated in the $x$-direction of the eigenvectors in terms of $U^{*}$. See [12] or Appendix A of [57] for the average state formula for the 3D non-conservative system (2). The $\bar{H}_{j+1 / 2}$ (involving the use of wavelet sensors and flux limiters) are also evaluated from the same average state. The dimension-by-dimension procedure of applying the approximate Riemann solver is adopted.

Denote the elements of the vector $\bar{H}_{j+1 / 2}$ by $\bar{h}_{j+1 / 2}^{l}, l=1,2, \ldots, 8$. The nonlinear portion of the filter $\bar{h}_{j+1 / 2}^{l}, l=1,2, \ldots, 8$, has the form

$$
\bar{h}_{j+1 / 2}^{l}=\frac{1}{2}\left(s^{N}\right)_{j+1 / 2}^{l}\left(\phi_{j+1 / 2}^{l}\right) .
$$

Here $\left(s^{N}\right)_{j+1 / 2}^{l}$ is the sensor to activate the higher order nonlinear numerical dissipation $\phi_{j+1 / 2}^{l}$. For example, $\left(s^{N}\right)_{j+1 / 2}^{l}$ is designed to be zero or near zero in regions of smooth flow and near one in regions with discontinuities. $\left(s^{N}\right)_{j+1 / 2}^{l}$ varies from one grid point to another and is obtained from a wavelet analysis of the flow solution [38]. The wavelet sensor can be obtained from the characteristic variables for each wave or a single sensor for all eight waves, based on pressure and density. Both methods were implemented but for the numerical tests in this paper, the simpler non-characteristic sensor was employed.

The dissipative portion of the nonlinear filter $\phi_{j+1 / 2}^{l}=g_{j+1 / 2}^{l}-b_{j+1 / 2}^{l}$ is the dissipative portion of a high order high-resolution shock-capturing scheme for the local $l$ th-characteristic wave. Here $g_{j+1 / 2}^{l}$ and $b_{j+1 / 2}^{l}$ are numerical fluxes of the uniformly high order high-resolution scheme and a high order central scheme for the $l$ th characteristic, respectively. It is noted that $b_{j+1 / 2}^{l}$ might not be unique since there is more than one way of obtaining $\phi_{j+1 / 2}^{l}$.

For the numerical examples, three forms of nonlinear dissipation $\phi_{j+1 / 2}^{l}$ were considered, namely:

- Dissipative portion of the fifth-order WENO scheme (WENO5) [59]. It can be obtained e.g., in the $x$-direction by taking the full WENO5 scheme in the $x$-direction and subtracting $D_{06} F_{j}$ (or $C_{06} F_{j}$ ).

- Dissipative portion of the a second-order MUSCL scheme [53].

- Dissipative portion of the Harten-Yee TVD scheme [53,57].

The nonlinear filter given by (8), if applied to the entire MHD system, will not preserve the divergence free magnetic field condition in general. For the computations in this paper and our previous work, the "No filter on B" option is chosen. That is, the nonlinear filter step (8) only applies to the first five equations of (1) or (2). Here, the complete set of eigenvalues and eigenvectors of the full symmetrizable MHD system is used to evaluate the first five equations of (1) or (2). With the divergence free spatial base scheme, the divergence free prop- 
erty should be preserved for uniform grids. Extensive grid convergence comparison of the "no filter on $B$ " with the "filter all of the MHD equations" (filter all) options were presented in [57]. Alternative approaches for obtaining divergence-free preserving shock-capturing filters follow in a similar vein as the constrained transport approach [8].

Note that if a high order linear filter step is employed prior to the nonlinear filter with the resulting solution denoted by $U^{* *}$ (right after the completion of a full time step of the base scheme step), it is understood that the numerical fluxes above are evaluated at $U^{* *}$ instead of $U^{*}$.

\subsubsection{Flow sensor by multiresolution wavelet analysis of the computed flow data}

The basic idea in obtaining the different flow sensors (e.g., $\left(s^{N}\right)_{j+1 / 2}^{l}$ ) by multiresolution wavelet analysis of the computed flow data can be found in Sjögreen and Yee [38] and Yee and Sjögreen [55]. Two types of multiresolution wavelets were considered. The mathematical procedures to obtain this type of flow sensor are very involved. However, the final algorithm is very simple. Interested readers are referred to the original papers for details. The two papers [15,37] are sources of background material for [38].

Wavelets were originally developed for feature extraction in image processing and for data compression. It is well known that the regularity of a function can be determined from its wavelet coefficients $[4,24,19]$ far better than from its Fourier coefficients. By computing wavelet coefficients (with a suitable set of wavelet basis functions), we obtain very precise information about the regularity of the function in question. This information is obtained just by analyzing a given grid function. No information about the particular problem which is solved is used. Thus, wavelet detectors are general, problem independent, and rest on a solid mathematical foundation.

As of the 1990s, wavelets have been a new class of basis functions that are finding use in analyzing and interpreting turbulence data from experiments. They also are used for analyzing the structure of turbulence from numerical data obtained from DNS or LES. See Farge [9] and Perrier et al. [31] for early work. There are several ways to introduce wavelets. One standard way is through the continuous wavelet transform and another is through multiresolution analysis, hereafter, referred to as wavelet based multiresolution analysis. Mallet and collaborators [19-24] established important wavelet theory through multiresolution analysis. See Refs. $[47,46]$ for an introduction to the concept of multiresolution analysis. Wavelet based multiresolution analysis has been used for grid adaptation [13], and to replace existing basis functions in constructing more accurate finite element methods. Here, we utilize wavelet based multiresolution analysis to adaptively control the amount of numerical dissipation.

Our wavelet flow sensor estimates the Lipschitz exponent of a grid function $f_{j}$ (e.g., the density and pressure). The Lipschitz exponent at a point $x$ is defined as the largest $\alpha$ satisfying

$$
\sup _{h \neq 0} \frac{|f(x+h)-f(x)|}{h^{\alpha}} \leqslant C,
$$

and this gives information about the regularity of the function $f$, where small $\alpha$ means poor regularity. For a $C^{1}$ wavelet function $\psi$ with compact support, $\alpha$ can be estimated from the wavelet coefficients, defined as

$$
w_{m, j}=\left\langle f, \psi_{m, j}\right\rangle=\int f(x) \psi_{m, j}(x) \mathrm{d} x,
$$

where

$$
\psi_{m, j}=2^{m} \psi\left(\frac{x-j}{2^{m}}\right)
$$

is the wavelet function $\psi_{m, j}$ on scale $m$ located at the point $j$ in space. This definition gives a so called redundant wavelet, which gives (under a few technical assumptions on $\psi$ ) a non-orthogonal basis for $L^{2}$. Theorem 9.2.2 in [4] states that if $\psi$ is $C^{1}$ and has compact support, and if the wavelet coefficients $\max _{j}\left|w_{m, j}\right|$ in a neighborhood of $j_{0}$ decay as $2^{m \alpha}$ as the scale is refined, then the grid function $f^{j}$ has Lipschitz exponent $\alpha$ at $j_{0}$. In practical computations, we have a smallest scale determined by the grid size. We evaluate $w_{m, j}$ on this scale, $m_{0}$, and a few coarser scales, $m_{0}+1, m_{0}+2$, and estimate the Lipschitz exponent at the point $j_{0}$ by a least square fit to the line [38] 


$$
\max _{j \text { near } j_{0}} \log _{2}\left|w_{m, j}\right|=m \alpha+c .
$$

Proper selection of the wavelet $\psi$ is very important for an accurate detection of the flow features. The result in $[24,23]$, which is used in [13], gives the condition that $\psi(x)$ should be the $k$ th-derivative of a smooth function $\eta(x)$ with the property

$$
\eta(x)>0, \quad \int \eta(x) \mathrm{d} x=1, \quad \lim _{x \rightarrow \pm \infty} \eta^{(k)}(x)=0 .
$$

Then the result is valid for $0<\alpha<k$. A continuous function $f(x)$ has a Lipschitz exponent $\alpha>0$. A bounded discontinuity (shock) has $\alpha=0$, and a Dirac function (local oscillation) has $\alpha=1$. Large values of $k$ can be used in turbulent flow so that large vortices or vortex sheets can be detected. Although the theorem above does not hold for $\alpha$ negative, a useful upper bound on $\alpha$ can be obtained from the wavelet coefficient estimate. The Appendix summarizes the wavelet flow sensor algorithm based on the Lipschitz exponent of a chosen computed flow vector(s). The remainder of this section gives a summary of the three basic steps described in [38] for obtaining the wavelet flow sensors.

\section{Step 1: Choose a wavelet type}

- Redundant form of Harten's multi-resolution form.

- 2nd-order B-splines.

- Wavelets that can distinquish high frequency oscillations from turbulence.

\section{Step 2: Choose flow variables to be sensed}

- Density andessure.

- Characteristic variables.

- Primitive or entropy variables.

\section{Step 3: Flow sensors}

- Apply wavelets to the flow variables to be sensed.

- Obtain the corresponding wavelet coefficient (involves 2-4 levels of nested difference operators).

- Obtain Lipschitz exponents (least square fit of the wavelet coefficients in domain of dependence).

- Determine the cutoff Lipschitz exponent (or a smooth transition).

- Use cutoff Lipschitz exponents as "flow sensors" (filter with appropriate numerical dissipations).

For example, a Lipschitz exponent with a value near zero, -1 , or wavelets with high order vanishing moments indicate of the presence of a discontinuity, spurious local high frequency oscillations or large vortices/vortex sheets respectively. For example, the flow sensor $\left(s^{N}\right)_{j+1 / 2}^{l}$ to turn on the shock-capturing dissipation using the cutoff procedure above is a vector (if applied dimension-by-dimension) consisting of "1's" and "0's".

\subsubsection{Proof of concept and related software development for multiscale flows}

During the last five years a highly parallel 3D Navier-Stokes/MHD computer code using the MPI library was built and has been well tested and debugged. The code contains high order compact and non-compact finite difference central base schemes with boundary modifications up to order 8 . It also contains a gradient-like ACM flow sensor and two multiresolution wavelet flow sensors. Three types of nonlinear filters and two types of high order linear filters (linear compact filters and non-compact linear filters) have been implemented into the code and have been well tested. The code also contains standard TVD, MUSCL, first and second-order Lax-Friedrichs, fifth-order WENO spatial schemes (WENO5) and explicit Runge-Kutta method of order one up to order four for method comparison. The proof of concept includes: 
- Numerical experiments on over three dozen representative test cases for inviscid and viscous 1D, 2D gas dynamics problems as well as ideal and non-ideal MHD test cases. These test cases range from simple 1D shock tube problems to multiscale and multiphysics problems. The majority of the test cases are either with exact, known converged solutions, or by 5-6 levels of grid refinement of known methods as reference solutions. Stability and accuracy of our filter schemes were then assessed according to these findings.

- Comparison among the 2nd-, 4th-, 6th- and 8th-order central base schemes.

- Comparison among different filter approaches with standard TVD, MUSCL and WEBO5 schemes.

- Comparison among the multistep filters and single step filters.

- Comparison among 6th-order central spatial base schemes with two different SBP boundary operators with the 6th-order compact spatial base scheme.

Studies show that our adaptive numerical dissipation control can accurately simulate a wide spectrum of flow speeds, flow types and governing equation sets, namely, from nearly incompressible to high speed shock/turbulence/combustion multiscale gas dynamics and MHD plasma flows. The filter scheme is more accurate and efficient than the standard structured or unstructured method commonly used in gas dynamics and plasma applications. In many instances, grid convergence was achieved by our high order filter schemes but not by standard second-order shock-capturing methods using the same grid sequence.

Using the same grid, more accurate solutions were obtained with our sixth-order filter schemes than with standard second-order shock capturing methods, which require similar CPU time, and a fifth-order weighted ENO scheme WENO5 [17], which is nearly three times as expensive. Results have been published in refereed journal articles and conference proceedings $[53,54,35,41,38,55,42,58,40,56,57,59,43,61,44,63]$. Interested readers are referred to these references for accuracy and stability studies of basic test cases. All of the examples shown below do not have exact solution or available experimental data. Unlike simple test cases, it is extremely difficult to measure certain error norm of the considered multiscale test cases. Assessment of the computed results are based on (a) many levels of grid refinement, (b) how well the fine scale feature of the flow are being resolved under grid refinement, and (c) by comparing with commonly used methods using the same grid sequence. It is in this spirit that the authors choose to compare among methods for the more difficult test cases shown below.

\section{Sample numerical results}

In this section, a combustion gas dynamics model, a Richtmyer-Meshkov instability for both the Euler and MHD systems, and three MHD numerical examples are selected to illustrate the performance of the scheme $[41,42,58,59,43,61,44,63]$. They are selected to show the: (a) capability of the filter scheme in simulating multiscale and multiphysics flows; (b) comparing among three different nonlinear filters; and (c) comparing between compact and non-compact base scheme; and (d) behavior of grid refinement on chaotic-like flows and shock-mixing problems. For the MHD results, all figures shown solve the conservative MHD system. In general, the computed results are slightly more accurate and stable than solving the symmetrizable system. For comparison between conservative and symmetrizable systems, see [57] for details.

The wavelet filter schemes using the dissipative portion of WENO5, second-order MUSCL and Harten-Yee TVD schemes with sixth-order spatial central base scheme $(d=0$ in (5)) for both the ideal and viscous nonideal MHD flux derivatives and a fourth-order Runge-Kutta method are denoted by WAV66weno5, WAV66mus and WAV66hy, respectively. The first number indicates the order of the base scheme for discretizing the inviscid flux derivatives. The second number indicates the order of the scheme for discretizing the viscous flux derivatives, if present. To adhere to the convention of previous work, even when dealing with inviscid flows, the same notation is used. Viscous flows are indicated with a non-zero Reynolds number. As mentioned before, there is no filtering for the viscous fluxes. If the coefficient $d \neq 0$ in the base scheme step (5) for approximating the inviscid flux derivative, the symbol "AD8" is added as in WAV66weno5+AD8. Computation using the same temporal and spatial scheme for the viscous MHD flux derivatives, and the standard fifth-order WENO scheme (using fourth-order Runge-Kutta temporal discretization) for the inviscid flux derivatives is denoted by WENO5. Computations using a second-order MUSCL and the Harten-Yee [57] TVD scheme for the inviscid MHD flux with the second-order central scheme for the viscous flux and a second-order 
Runge-Kutta method are denoted by MUSCL and HY, respectively. Unless otherwise indicated, the notation convention "WAV66" here refers to WAV66 (ACM66) with any of the three nonlinear filters discussed above. Scheme ACM66 is the same as WAV66 except the ACM sensor is employed.

The entropy fix parameter $\epsilon$ is 0.25 [16,52] for the Harten-Yee, MUSCL, WAV66mus and WAV66hy schemes (to avoid expansion shocks and carbuncle phenomenon). The entropy value for the degenerate zero eigenvalue of the conservative system is in the range of $10^{-7}$ to $10^{-10}$. The cutoff wavelet Lipschitz exponent $\beta$ is 0.5 [38] for all the wavelet filter schemes. See [53,38,55] or Appendix B of [57] for the definition of $\epsilon$ and $\beta$. Except for WENO5, the van Leer version of the van Albada limiter is used. For the second-order MUSCL scheme, the limiter is applied to the primitive variables. All methods employed the Roe's approximate Riemann solver for the gas dynamics cases and the Gallice approximate Riemann solver for the MHD cases using our method of solving the conservative MHD system [57]. The following illustrates the performance of the two base schemes (5) and (6), and the three different nonlinear filters solving the conservative MHD.

\subsection{A viscous combustion model with multi-chemical reactions}

The first example is the same viscous reactive flow considered in [6,7]. It consists of a planar Mach 2 shock in air interacting with one and two circular zones of hydrogen bubbles. The governing equations are the compressible Navier-Stokes equations with four species undergoing multi-chemical reactions. The chemical reaction is modeled by a single-step reversible reaction using $\mathrm{H}^{2}, \mathrm{O}^{2}, \mathrm{H}^{2} \mathrm{O}$ and $\mathrm{N}^{2}$. A Prandtl number, $P r=0.72$, Schmidt number $S c=0.22$, and the perfect gas equation of state approximation are used. The mixture specific heat at constant pressure was obtained from McBride et al. [25]. The Svehla [48] species viscosity constants and the Wilke's law model [51] for the mixture viscosity are used. The temperature of the hydrogen and air in the undisturbed region ahead of the shock is set to $1000{ }^{\circ} \mathrm{C}$ with a pressure of $1 \mathrm{~atm}$. and zero velocity. A Mach 2 shock is placed at $x_{\mathrm{s}}=0.005$. The gradient in pressure across the shock in conjunction with the gradient in fluid density between the air and hydrogen produces a large increase in vorticity as the shock passes through the hydrogen fuel. This can be seen in the earlier spectral numerical simulation of Don and Quillen [6] and Don and Gottlieb [7] (see Fig. 1c). Their high order spectral shock filter scheme is not able to suppress the spurious oscillations due to the spectral discretization. This is a CPU intensive and very stiff viscous test case with no experimental data available. A variation of a similar problem has been investigated by various computational researchers. With a few levels of grid refinement, most numerical methods are able to capture the global features of the evolving bubbles but not the fine scale structure inside the bubbles. The situation is compounded by the fact that as the grid is refined, the interplay of different stiffness (e.g., viscous terms, combustion model and fine grid spacing induce stiffness) impose an unreasonable time step constraint on the simulation. What is discussed and shown below illustrates the high accuracy of the filter scheme comparing with TVD, MUSCL, WENO5, and a very fine grid as the reference solution.

Extensive grid convergence studies using WAV66 and ACM66 were conducted in Sjögreen and Yee [42] on this viscous supersonic reactive flow. For this problem, more accurate solutions were obtained with WAV66
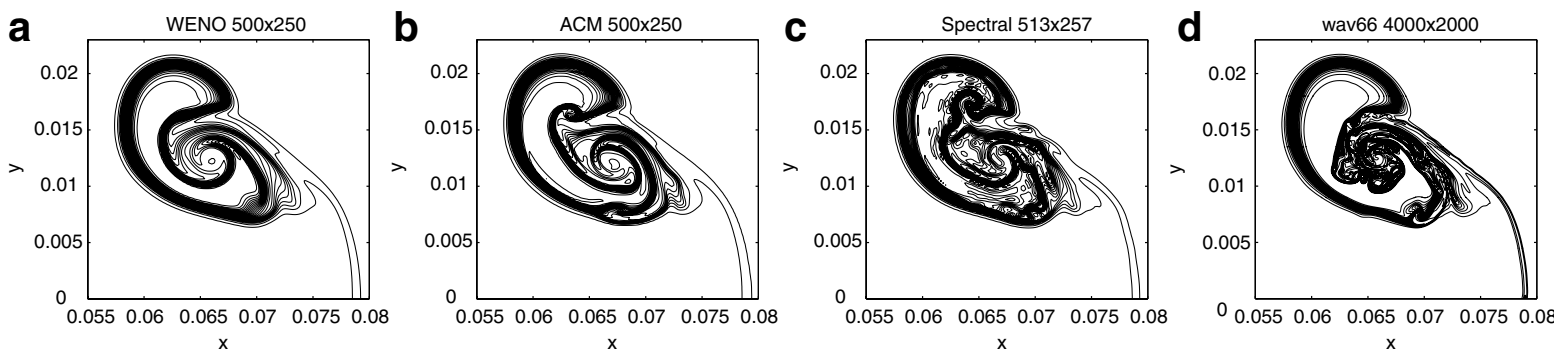

Fig. 1. Comparison of the hydrogen mass fraction contours at time $60 \mu$ s. (a) WENO5, $500 \times 250$. (b) ACM66, 500 $\times 250$. (c) Spectral with filter, $513 \times 257$. (d) WAV66, $4000 \times 2000$. 
and ACM66 than with MUSCL and TVD, which is of similar CPU cost, WENO5, which is nearly three times as expensive, and the spectral shock filter scheme, which is more expensive. Fig. 1 shows the comparison among the three methods with a spectral shock filter scheme for a single zone of hydrogen bubbles. The solution by WAV66-RK4 using a $4000 \times 2000$ grid is used as the reference solution (Fig. 1d). Using the same grid, WAV66 and ACM66 can resolve the fine scale structure inside the bubble better than MUSCL, TVD and WENO5. The resolution of WAV66 using a $500 \times 250$ grid is similar to that of ACM66 (Fig. 1b).

\subsection{Comparison among three nonlinear filters [59]}

Comparison among the three nonlinear filters discussed previously using the non-compact 6th-order spatial base scheme can be found in Yee and Sjogreen [59]. Here only the 2D compressible Orszag-Tang vortex problem [3] consisting of periodic boundary conditions and smooth initial data is considered

$$
\left(\rho, u, v, w, p, B_{x}, B_{y}, B_{z}\right)=(25 / 9,-\sin y, \sin x, 0,5 / 3,-\sin y, \sin 2 x, 0) .
$$

The computational domain is $0<x<2 \pi, 0<y<2 \pi$ and the computation stops at time $T=3.14(\approx \pi)$, when complicated structure and discontinuities have formed and interacted. The comparison among the three filter schemes (no filter on B option), WENO5, MUSCL and Harten-Yee (HY) using six uniform $51 \times 51$, $101 \times 101, \quad 201 \times 201,401 \times 401,801 \times 801$ and $1601 \times 1601$ grids for ideal and non-ideal MHD were conducted.

Grid convergence was obtained by all six methods (WENO5, MUSCL, HY, WAV66weno5, WAV66mus and WAV66hy) using the $801 \times 801$ grid. Computations based on a $1601 \times 1601$ grid are used as the reference solutions. For $51 \times 51$ through $401 \times 401$ grids, small structures are better captured by the three filter methods than by WENO5, MUSCL or Harten-Yee (see e.g., $2<x<4,0<y<1$ of Fig. 2). In addition, for the inviscid case, the three filter methods are more stable than the other three methods in the sense that a larger CFL number can be used. Fig. 2 shows the computations using a CFL of 0.6 and an $801 \times 801$ grid. WENO5 and MUSCL show a slight small oscillation. These oscillations can be suppressed by applying the limiter to the characteristic variables in the MUSCL scheme (figures not shown).

For the viscous case, the flow structure is less complicated than the inviscid case. All computations use a CFL of 0.6. For coarse grids, again small structures are better captured by the three filter methods than by WENO5, MUSCL or Harten-Yee. In other words, the three filter methods exhibit similar accuracy as the three standard shock-capturings methods with a coarser grid. For both the inviscid and viscous computations, all three filter methods using the "no filter on $B$ " option are divergence-free preserving, whereas the "filter all" option as well as standard WENO5, MUSCL and HY without divergence cleaning are not divergence free. Their $\nabla \cdot \mathbf{B}$ numerical error at $T=3.14$ increases as the grid is refined. See [57] for some illustrations.

Among the various test cases conducted in [59], MUSCL and Harten-Yee require similar CPU time. The CPU time required by the three filter methods is within $15 \%$ of one another depending on the problem, grid spacings and time steps. They require slightly more CPU time $(20 \%)$ than the Harten-Yee and MUSCL schemes. This is due to the fact that all filter schemes require only one Riemann solve per time step per direction (independent of the time discretizations of the base scheme step) as opposed to two Riemann solves per time step per direction by the MUSCL and Harten-Yee schemes using a second-order Runge-Kutta method. WENO5 requires at least twice the CPU time of all other methods since four Riemann solves per time step per direction are required by WENO5-RK4.

\subsection{Compact vs. non-compact base scheme comparison [61]}

Numerical experiments on the performance of compact and non-compact sixth-order spatial base schemes were conducted on the test cases studied in $[57,44,63]$. The results are reported in $[61,44,63]$. Only the KelvinHelmholtz and the compressible version of the Orszag-Tang problems are summarized here. For the KelvinHelmholtz problem, the computations stop at an evolution time $T=0.5$ when the solution is still smooth enough that it can be solved by the base scheme alone in conjunction with a small amount of linear dissipation in (5). For the compact base scheme (6) with $d=0$, a compact linear filter is needed. All methods considered use uniform grid spacing. 


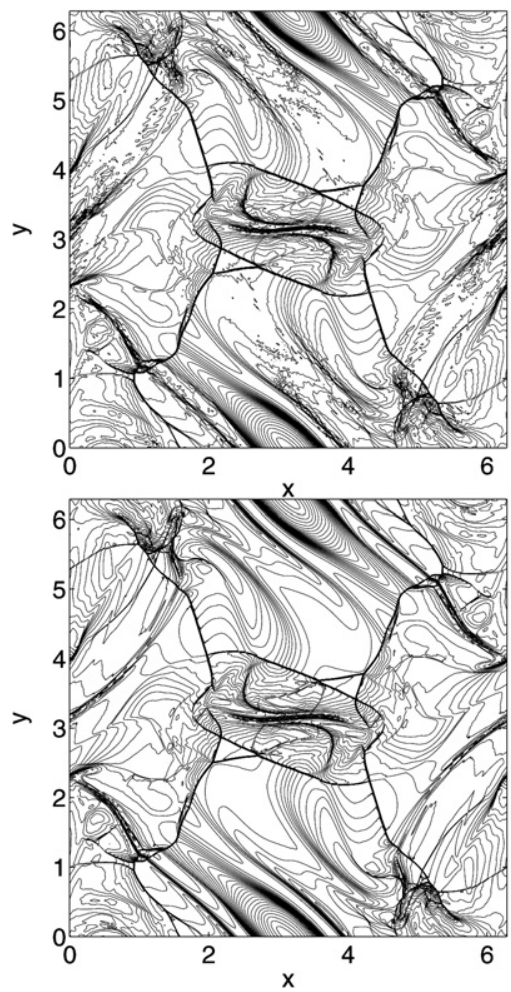

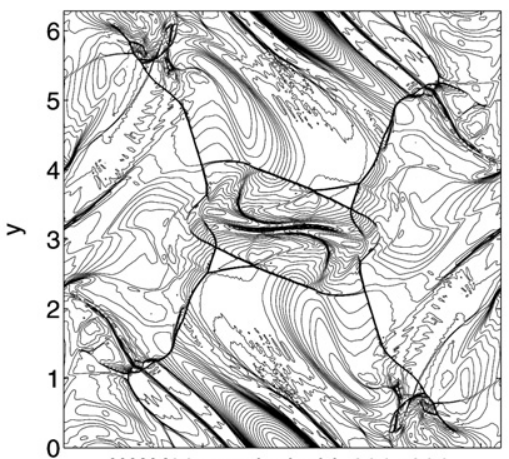

WAV66mus, inviscid, $801 \times 801$

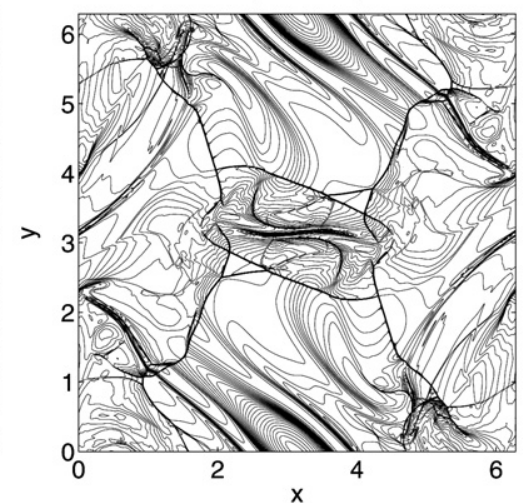

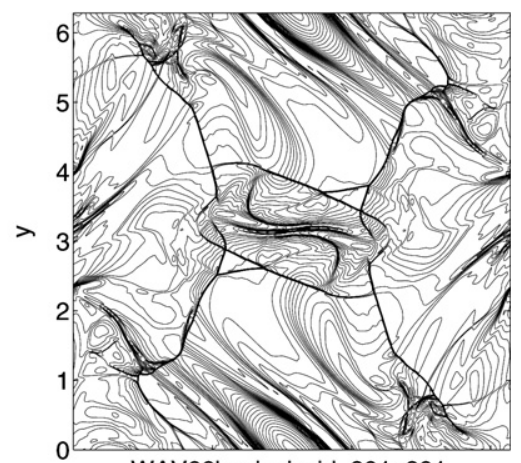

WAV66hy, inviscid, $801 \times 801$

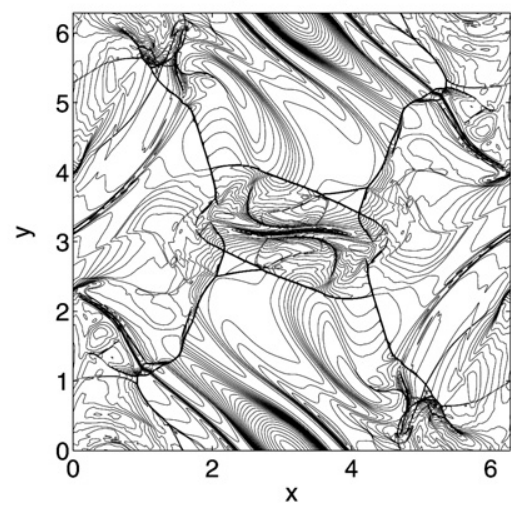

Fig. 2. Inviscid Orszag-Tang problem using a $801 \times 801$ grid. Density contours for ideal MHD at $T=3.14$. Top row: WENO5 (left), MUSCL (middle), HY (right). Bottom row: WAV66weno5 (left), WAV66mus (middle), WAV66hy (right).

Computations by the sixth-order centered compact base scheme (6) with $d=0$ in conjunction with the compact linear filter [10], denoted by COMP66+COMPfi using five grids $51 \times 101,101 \times 201,201 \times 401,401 \times 801$ and $801 \times 1601$ are compared. The accuracy using (6) with $d=0.0005$ and without COMPfi (i.e., COMP66+AD8) is similar. The same computations using the sixth-order central scheme (5) with $d=0.0005$, denoted by CEN66+AD8, were conducted. As a reference solution, computations using the eigth-order central scheme with the 10th-order linear dissipation and a dissipation coefficient of 0.0005 as the base scheme, denoted by CEN88+AD10, for the same six grids were used. There is no visible difference in accuracy between COMP66+COMPfi and CEN66+AD8. Similar accuracy was obtained using either (5) or (6) as the base scheme in conjunction with any of the nonlinear filters discussed above. Fig. 3 shows a comparison.

For the Orszag-Tang problem, after shock waves develop, the use of the base scheme alone is not sufficient to obtain a stable solution. The computations stop at an evolution time $T=3.14$. The compact base scheme in conjunction with the compact linear filter also becomes highly oscillatory. The left and middle columns of Fig. 4 show the computations by, respectively, (a) a two-step filter, COMP66+COMPfi in conjunction with the WENO5 filter (WENOfi) denoted by COMP66+COMPfi+WENOfi, and (b) a one-step filter, COMPP66+WENOfi (compact base scheme in conjunction with the nonlinear filter only). The right column of Fig. 4 shows the same computation using CEN66+WENOfi. The small spurious oscillations by CEN66+WENOfi using the $101 \times 101$ grid can be suppressed by adding the AD 8 term to the central base scheme with $d=0.0001$. The change of the notation from WAV66weno5 to CEN66+WENOfi, e.g., is to distinguish the two spatial base schemes. As the grid is refined $(201 \times 201$ or larger $)$, these small spurious oscillations vanished by CEN66+WENOfi alone without the aid of AD8. For finer grids $(201 \times 201$ or larger $)$, the numerical solutions exhibit spurious oscillations by COMP66+WENOfi but not by CEN66+WENOfi (same as WAV66weno5).

These spurious oscillations become more pronounced as the grid is refined. Unlike the central base scheme, it appears that the compact base scheme with the nonlinear filter alone is not able to suppress the spurious 

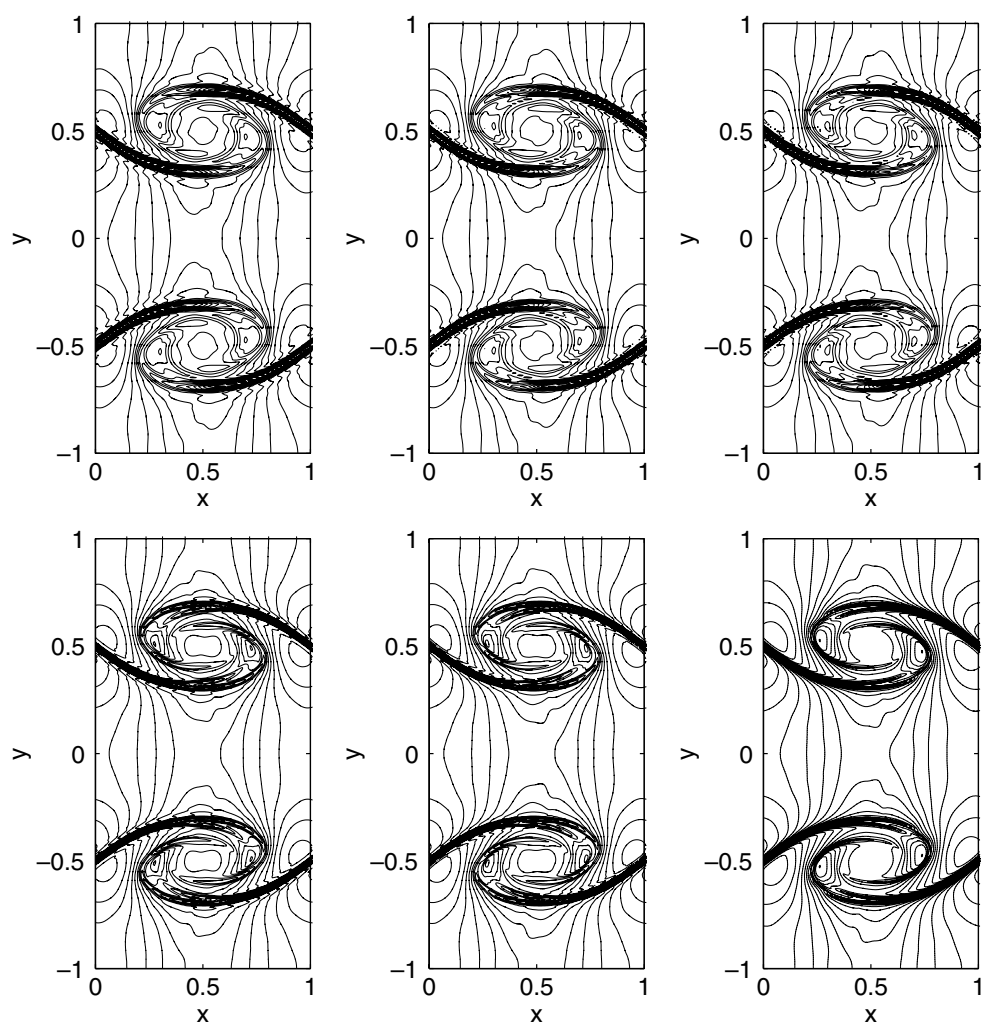

Fig. 3. Density contours of the Kelvin-Helmholtz problem at $T=0.5$ using $101 \times 201$ (top) and $201 \times 401$ (bottom) grids by COMP66+COMPfi (left) and CEN66+AD8 (middle) compared with reference solution by CEN88+AD10 (right) using $101 \times 201$ and $801 \times 1601$ grids.

oscillation completely as the grid is refined. It needs the combination of the compact linear filter and nonlinear filter (or by adding AD8 as part of the compact base scheme step) in order to suppress the spurious oscillations. If we add $d=0.0005$ in (b) above as part of the base scheme step, there is no visible difference in accuracy among the three methods for grids $201 \times 201$ or larger. (i.e., comparing COMP66+COMPfi+WENOfi, COMP66+AD8+WENOfi and CEN66+WENOfi). See [57] for the reference solution. The same comparison was performed on other test cases studied in $[57,44,63]$ with and without physical viscosity and resistivity, and the same conclusion was arrived at as in the aforementioned two test cases. Our recent gas dynamics and MHD studies (see also the next example) arrive at the same conclusion drawn in [53] on the behavior of compact schemes for problems containing multiscale shock interaction for the gas dynamics case.

High order compact schemes are methods of choice for many incompressible and low speed turbulent/ acoustic flows due to their advantage of requiring very low number of grid points per wavelength. In the presence of multiscale shock interactions, however, this desired property of high order compact base schemes seems to have diminished in both the gas dynamic and MHD test cases that we have studied (compared with the same order of accuracy of non-compact central base schemes). Also the compact spatial base scheme requires more CPU time per time step and it is less compatible with parallel computations than the central spatial base scheme. Consequently, the compact spatial base scheme requires added CPU time in a parallel computer framework.

\subsection{Richtmyer-Meshkov instability (RMI) [44,63]}

This study illustrates many aspects of the interplay between multiscale and multiphysics flows with numerical simulations, e.g., the suppression of the RMI in the presence of a magnetic field, and the failure of grid 

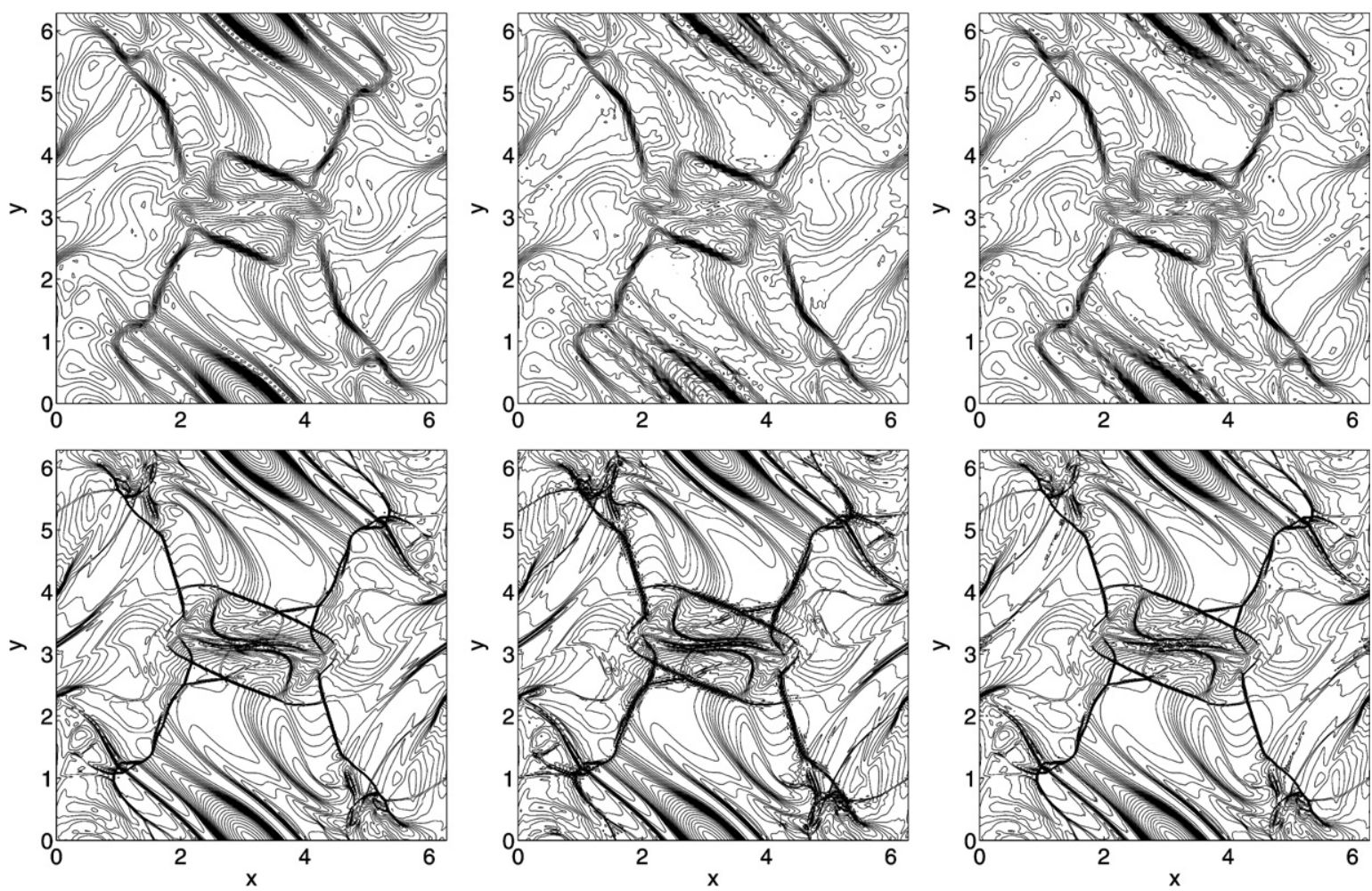

Fig. 4. Density contours of the Orszag-Tang problem at $T=3.14$ using $101 \times 101$ (top) and $401 \times 401$ (bottom) grids by COMP66+COMPfi+WENOfi (left), COMP66+WENOfi (middle) and CEN66+WENOfi (right).

\section{Richtmyer Meshkov Instability (RMI)}

(Suppression of RMI in the Presence of a Magnetic Field)

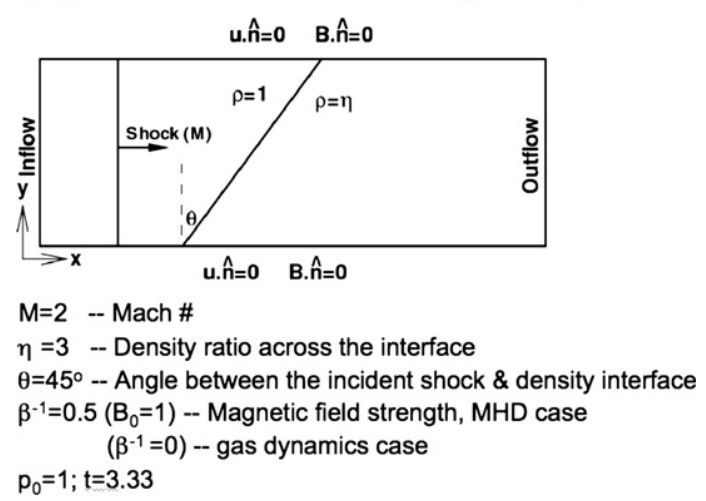

Fig. 5. Problem definition.

refinement for unsteady chaotic-like inviscid flows. RMI occurs when an incident shock accelerates an interface between two fluids of different densities. This interfacial instability was theoretically predicted by Richtmyer [34] and experimentally observed by Meshkov [26]. For the present study, the RMI problem investigated by Samtaney [27] and Wheatley et al. [50] as indicated in Fig. 5 has been chosen. The mathematical models are the 2D Euler gas dynamics equations and the ideal MHD equations. The computational domain is $-2<x<6$ and $0<y<1$. A planar shock at $x=-0.2$ is moving (left to right) toward the density interface with an incline angle of $\theta$ with the lower end initialized at $x=0$. The density ratio across the interface 
is denoted by $\eta$, and the nondimensional strength of the magnetic field $\beta=2 p_{0} / B_{0}^{2}$, where the initial pressure in the preshocked regions is $p_{0}=1$, and $B_{0}$ is the initial magnetic field. The initial magnetic field is uniform in the $(x, y)$ plane and perpendicular to the incident shock front. Numerical results shown below are for $M=2$, $\theta=45^{\circ}, \eta=3, \beta^{-1}=0$ (Euler gas dynamics) and $\beta^{-1}=0.5$ (magnetic field present). The computation stops at an evolution time $t=3.33$. For this set of parameters and all studied numerical schemes, instability occurs near $t=1.8$ for the gas dynamics case but not for the MHD case for the entire time evolution. Our numerical results exhibit behavior similar to the study of Samtaney.

Computations by COMP66+COMPfi+WENOfi using a $801 \times 101$ grid are shown in Fig. 6 (left) for the inviscid gas dynamics (GD) and the ideal MHD equations. The same computation using CEN66+WENOfi (WAV66weno5) is shown in Fig. 6 (right). For this low resolution grid, the accuracy between the two filter methods is similar. See Fig. 7 for the grid refinement study below. Computations using COMP66+WENOfi (i.e., without the linear compact filter step) or COMP66+COMPfii (i.e., without the nonlinear WENOfi filter step) indicate spurious oscillations around shock regions. The numerical example arrives, again, at the same conclusion drawn in $[53,61]$ on the behavior of compact spatial schemes for problems containing multiscale shock interaction.

Fig. 7 shows the inviscid gas dynamics comparison among a second-order MUSCL, CEN66+MUSfi ((WAV66mus): sixth-order filter using the dissipative portion of MUSCL as part of the nonlinear filter), and CEN66+WENOfi and for four grids $(801 \times 101,1601 \times 201,3201 \times 401,6401 \times 801)$. Not shown is the same computation using CEN66+HYfi (WAV66hy). For similar resolution, the standard shock-capturing

\section{6th-Order Compact vs. Central Spatial Base Schemes Euler \& MHD, (801x101)}

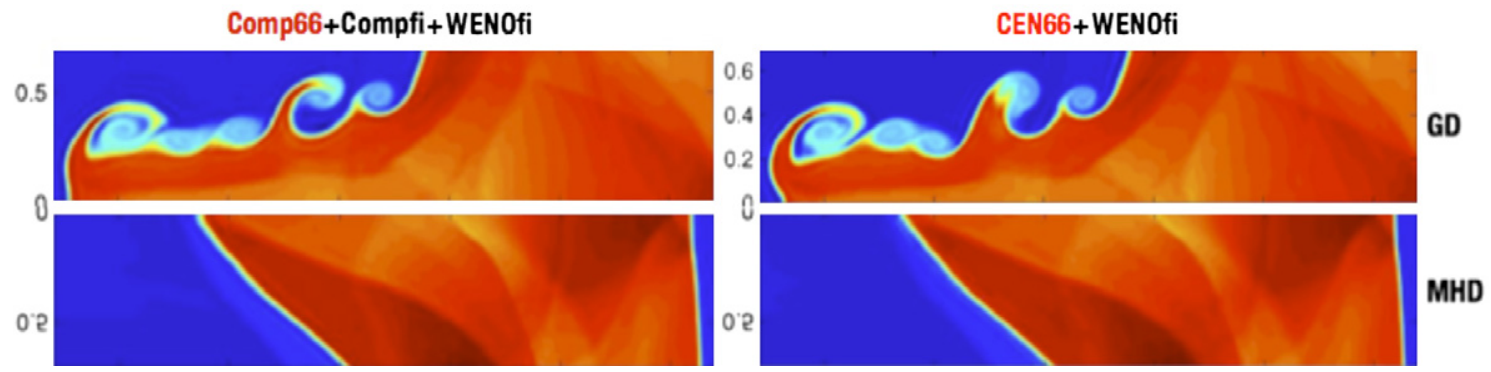

Fig. 6. RMI problem. Comparison between Euler gas dynamics and MHD for the sixth-order compact spatial base scheme (left) and the sixth-order central (non-compact) spatial base scheme (right) using a $(801 \times 101)$ grid at $t=3.33$. MHD solutions shown are mirror images of the original computations.

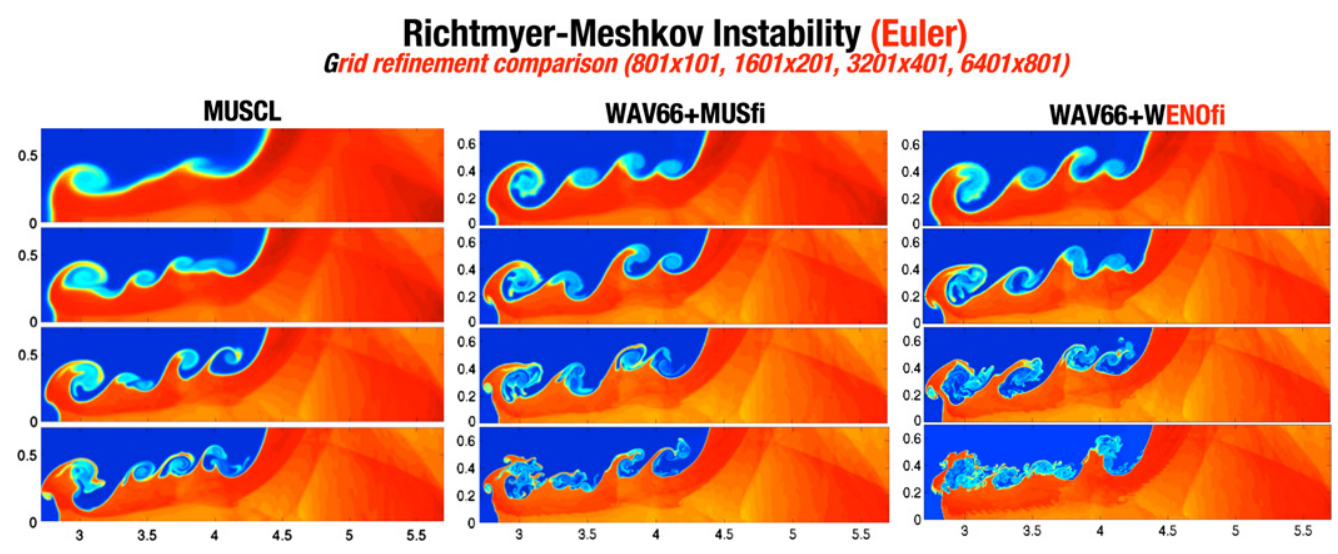

Fig. 7. RMI problem. Grid refinement study of the second-order MUSCL (left), CEN66+MUSfi (WAV66mus; middle) and CEN66+WENOfi (WAV66weno5; right) at $t=3.33$ using $(801 \times 101),(1601 \times 201),(3201 \times 401)$ and $(6401 \times 801)$ grids . 
scheme MUSCL requires nearly three times finer grid size per spatial direction than CEN66+MUSfi, CEN66+HYfi and CEN66+WENOfi. The eddy structures are different among the three filter methods and they are very different from the Samtaney adaptive mesh refinement (AMR) simulation with an equivalent uniform grid of $16,384 \times 2048$.

The effect of high order linear dissipation added to the base scheme in conjunction with nonlinear filters for the RMI is reported in [63]. Fig. 8 shows the effect of linear dissipation (AD8) added to the base scheme in conjunction with two different filters CEN66+AD8+WENOfi (WAV66weno5+AD8) and CEN66+AD8+HYfi (WAV66hy+AD8) for four linear dissipation coefficients of AD8 (0, 0.0005, 0.001, 0.002) in (5) using a $(6401 \times 801)$ grid. The top sub-figures show the computations using only a nonlinear filter $($ AD $8=0)$. The rest of the sub-figures are computations using three different non-zero AD8 coefficients. With such a fine grid, the eddy structures are very different. Traditionally, when dealing with non-chaotic turbulent type flows, grid refinement can serve as a measure of the accuracy and convergence property of the numerical methods. However, due to the chaotic-like nature of the present Euler MRI, the small amount of high order linear dissipation present on the spatial base scheme actually alters the type of governing equation that we are solving. In effect, we are solving the Navier-Stokes equations with a linear viscosity term. This in conjunction with the adaptive nonlinear filter (i.e., shock-capturing dissipations were employed at locations that are dictated by the wavelet flow sensor), results in a complex interplay of different types and amount of numerical dissipation which can alter the chaotic pattern of the flow. The study can serve as a good example of failure of grid refinement for unsteady chaotic-like inviscid flow. As the grid is refined (in conjunction with different amounts and types of numerical dissipations contained in each scheme), smaller and smaller eddies are formed which combine to affect global flow through the energy cascade effect. Note that for the inviscid RMI, WAV66weno5+AD8 requires nearly twice the CPU time than that of WAV66hy and WAV66mus.

For Navier-Stokes computations with Reynolds numbers higher than 10,000, same failure of grid refinement was encountered by all studied methods. In the presence of physical viscosity and for Reynolds number below 10,000, grid refinement has been achieved by all studied methods. To achieve similar resolution, MUSCL and WENO5 required more than double the grid points in each spatial direction than that of filter schemes and yet the CPU time per grid point and time step with the same grid for most of the studied methods is comparable. These results, including physical viscosity effects, are reported in $[44,63]$. Future work, includ-

\section{Effects of Linear Dissipation \& Nonlinear Filters Euler RMI, AD8 $=(0,0.0005,0.001,0.002), 6401 \times 801$}
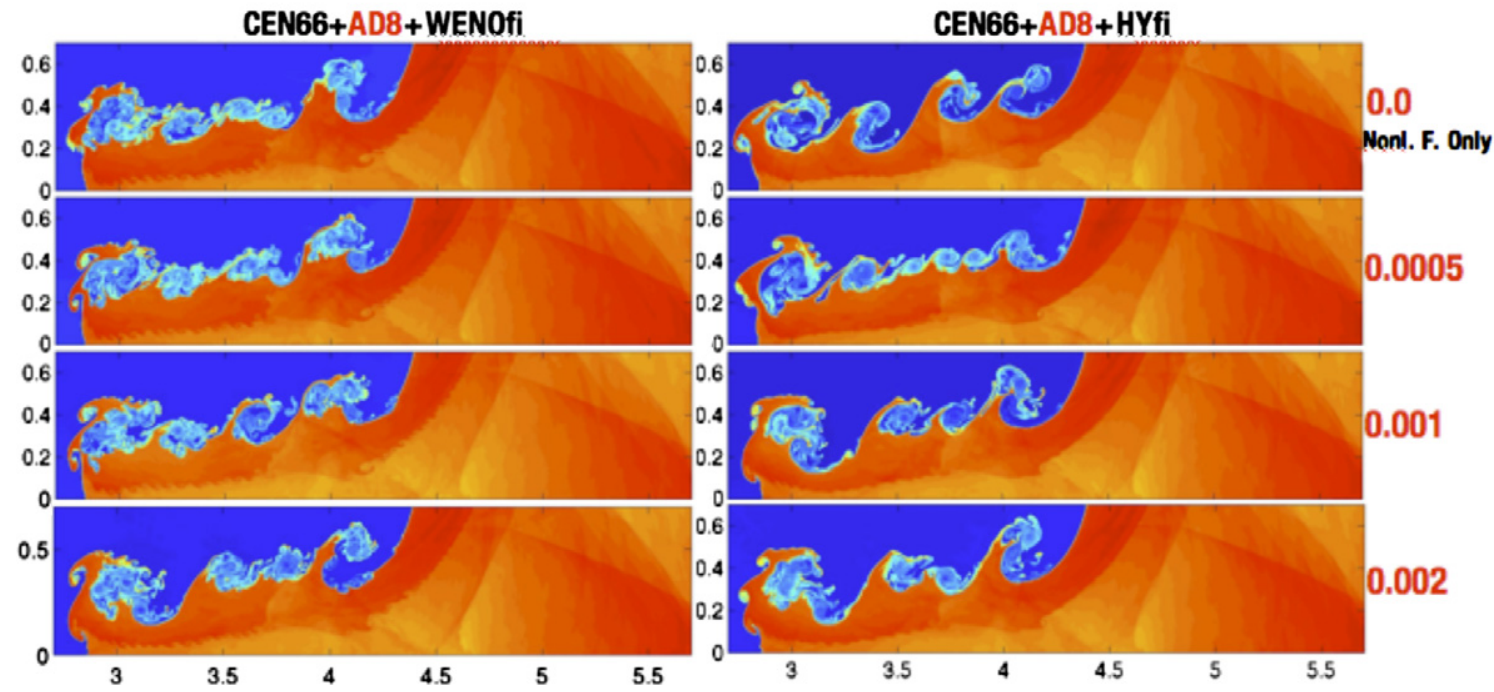

Fig. 8. RMI problem. Effect of linear dissipation (AD8) and nonlinear filter by two different filters and AD8 coefficients of (0, 0.0005 , $0.001,0.002)$ using a $(6401 \times 801)$ grid. 


\section{Vortex Pairing in a Time-Developing Mixing Layer \\ $\left(M_{c}=0.8, R e=1000, T_{R}=300 K\right.$, Prandtl $\left.\#=0.72\right)$}

Normalized with vorticity thickness:

$$
\delta_{\omega}=\frac{u_{1}-u_{2}}{(d u / d y)_{\max }}
$$

T \& c: determined by assuming constant stagnation enthalpy

IC:

$$
u_{1}=1, u_{2}=-1, T_{1}=T_{2} \text {, }
$$

Inital shear profile: $u=0.5 \tanh (2 y)$

Crocco-Busemann:

$$
c^{2}=c_{1}^{2}+\frac{\gamma-1}{2}\left(u_{1}^{2}-u^{2}\right)
$$

Initial perturbations:

$$
v^{\prime}=\sum_{k=1}^{2} a_{k} \cos \left(2 \pi k x / L_{x}+\phi_{k}\right) e^{\left(-y^{2} / b\right)}
$$

$$
\begin{aligned}
& L_{1}=30, b=10 \\
& a_{2}=0.05, \phi_{2}=-\pi / 2 \\
& a_{1}=0.01, \phi_{1}=-\pi / 2
\end{aligned}
$$

$$
\begin{aligned}
& \text { BC: Periodic in } x \text {, slip walls in } y \\
& \text { Grids: } \\
& \quad y=\frac{L_{y}}{2} \frac{\sinh \left(b_{y} \eta\right)}{\sinh \left(b_{y}\right)}, \quad L_{y}=100, b_{y}=3.4 \\
& \text { uniform in } x
\end{aligned}
$$

Fig. 9. Vortex pairing in time-developing mixing layer gas dynamics problem.

ing the bracketing of the bifurcation Reynolds number for the Navier-Stokes system where the RichtmyerMeshkov instability ceases to exist is planned (see Fig. 9).

\subsection{Vortex pairing in time-developing mixing layer result [60,43]}

The fourth numerical example reported in $[60,43]$ is a viscous mixing layer problem. Fig. 9 shows the schematic of the flow condition of the gas dynamics model studies in [53]. For the magnetized case, in addition to the gas dynamics initial conditions (ICs) and boundary conditions (BCs) indicated in Fig. 9, the initial magnetic field is 0.1 in the $x$-direction and zero in the other two directions. The Reynolds number of $R e=1000$, the conductivity coefficient of 100 and the Prantdl number of 0.72 are used for this test case. A grid mildly stretched in the $y$-direction and uniform in the $x$-direction is used. See Yee et al. [53] for the basic gas dynamic flow set up and the performance of WAV66 and AMC66. Fig. 10 shows the comparison of WAV66 and TVD22 (a second-order TVD scheme). Without the magnetic effect, the mixing and shock/shear patterns are more complicated. With a shear initial magnetic profile, the mixing is greatly diminished. Our gas dynamics and MHD comparison illustrates the possible taming of the turbulence effect by plasma injection. Although WAV66 and TVD22 require the same CPU time, WAV66 only requires 1/4 of the grid points in each spatial direction for the same numerical resolution as TVD22.

Fig. 11 shows the comparison of our ACM66 scheme with MUSCL for ideal MHD computations. ACM66 required at least 50\% fewer grid points per direction with similar resolution as MUSCL and WENO5 (figures not shown; see [60,43]). Comparison of ideal MHD with viscous resistive MHD for $R e=10^{3}$ with five different conductivities $\sigma=10^{6}, 10^{4}, 10^{3}, 100$ and 50 at time $T=90$ can be found in [43]. The ACM66 solution converges for the viscous resistive model for $\sigma=10^{6}, 10^{5}, 10^{3}, 100$ and 50 using grid sizes $1601 \times 1601$, $1601 \times 1601,801 \times 801,201 \times 201$ and $201 \times 201$, respectively. For ideal MHD, the fine scale structures are quite well resolved using a $1601 \times 1601$ grid. However, grid convergence has not been obtained for this case. Our studies indicated that lower accuracy by diffusive shock-capturing schemes such as MUSCL for the ideal MHD is similar to that of the solution with added $R e=1000$ and $\sigma=10^{3}$ by ACM66. See Figs. $6 \mathrm{c}$ and $7 \mathrm{~d}$ in [43]. The resolutios of WENO5 and Harten-Yee TVD schemes exhibit a similar diffusive pattern as MUSCL.

For the comparison of viscous resistive MHD for $R e=10^{8}$ with six conductivity coefficients $\sigma=10^{6}, 10^{4}$, $10^{3}, 10^{2}, 50$ and 10 at $T=90$, see [60,43]. The ACM66 solution converges for $\sigma=10^{3}, 100$ and 50 using a grid 


\section{MHD Vortex Pairing in Time-Developing Mixing Layer Re=1000, Resis. Coeff: $=100$ $T=120$, WAV66 vs. TVD22}
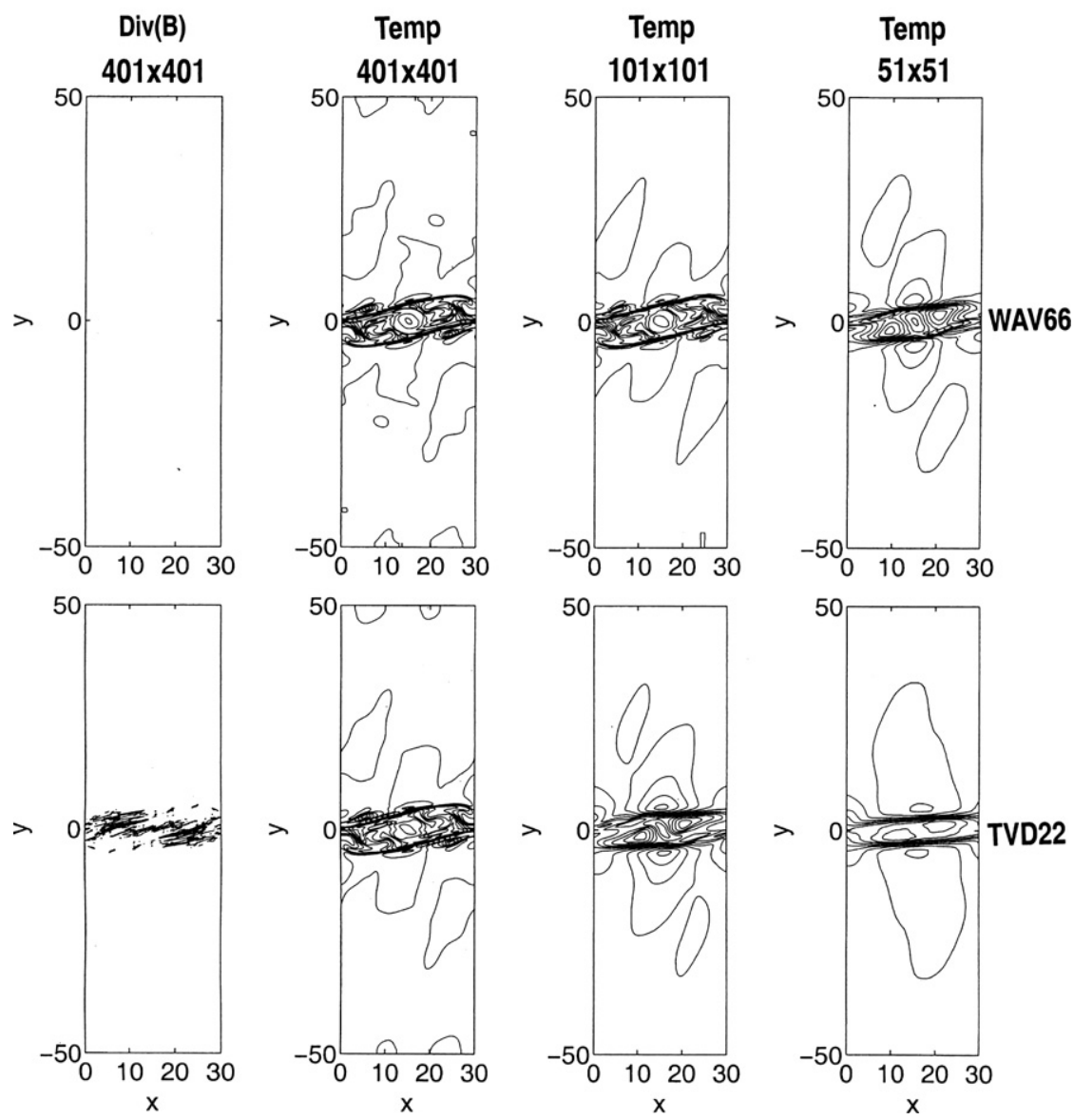

Fig. 10. MHD vortex pairing in time-developing mixing layer problem: Comparison between second-order TVD scheme (TVD22) and WAV66.

of $1601 \times 1601,801 \times 801$ and $401 \times 401$, respectively. For the three higher $\sigma=10^{6}, 10^{5}, 10^{3}$, the fine scale structures are quite well resolved using a $1601 \times 1601$ grid. However, grid convergence has not been obtained for these three $\sigma$ values on the very fine scale structures of the flow.

The study in $[60,43]$ also gives some insights on the effect of the resistive and viscous terms compared with ideal MHD. The most interesting result is that without adaptive numerical dissipation control, the commonly used shock-capturing schemes such as MUSCL, TVD and WENO5 solving ideal MHD produce solutions as if added physical dissipation were present.

With the Hall term included, for small Hall coefficient $\beta_{h}<0.2$ there is not much effect on the overall flow structure over the pure resistive MHD with the same $R e$ and plasma $\beta_{p}$. As the Hall coefficient is increased beyond 0.2 , the problem becomes more stiff and the computation using our filter scheme is stable only with grids that are smaller than $801 \times 801$. Although the more diffusive MUSCL and Harten-Yee are stable for a denser grid, the resolution is similar to the coarser grid solution by the filter method. Fig. 12 shows three computations with different Hall coefficient $\beta_{h}$ and conductivity coefficient $\sigma$. With the studied flow parameters, the Hall MHD flow patterns deviate slightly from the resistive MHD. For larger $\beta_{h}$, all considered methods become unstable even with smaller $R e$ and $\sigma$ value $s$.

Our preliminary study in [43] on two blunt body test cases shows the effect of the resistive and Hall coefficients on the flow structures compared with ideal MHD. The result of the two blunt body problems indicate 
a

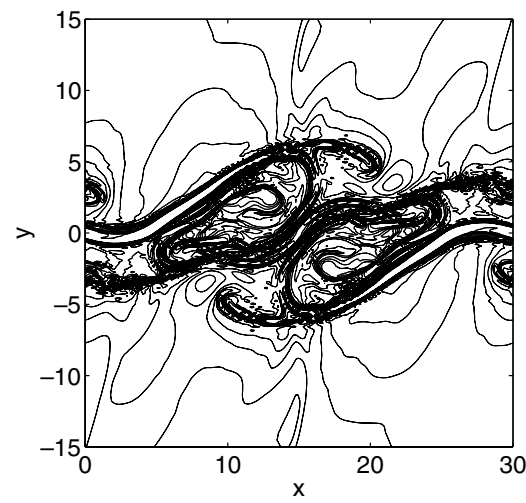

C

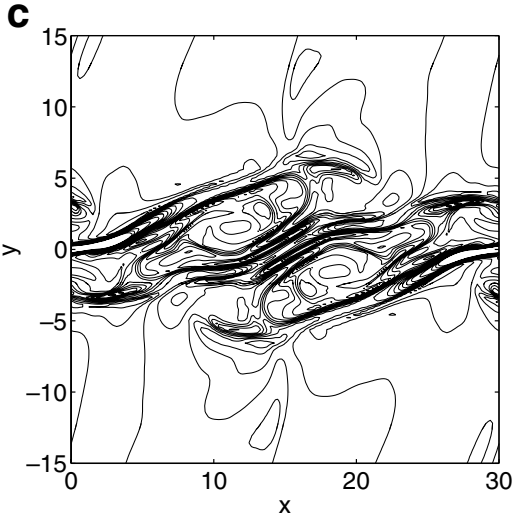

b

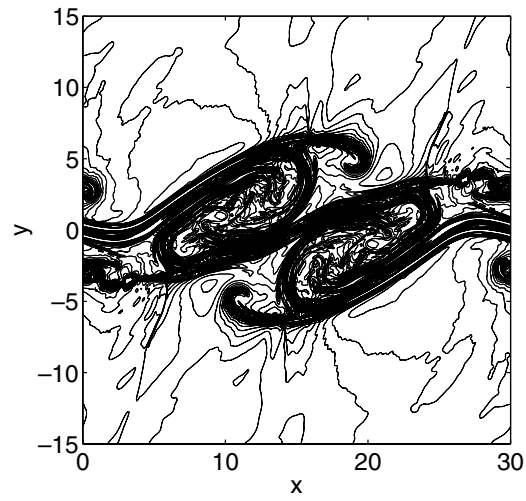

d

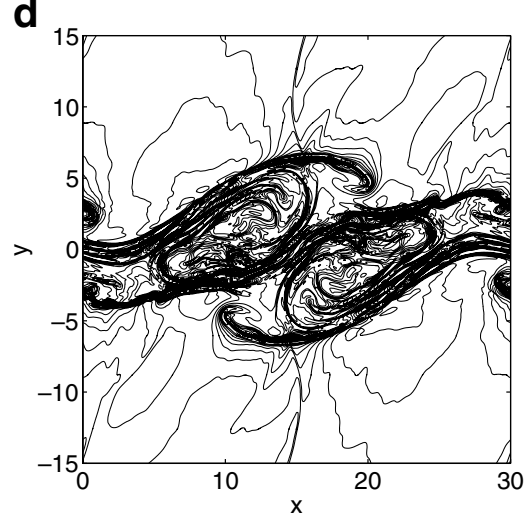

Fig. 11. Comparison of the temperature contours of ACM66 (top row) with MUSCL (bottom row) at $T=90$ for ideal MHD using $201 \times 201$ and $801 \times 801$ grids. (a) ACM66, $201 \times 201$ (b) ACM66, $801 \times 801$ (c) MUSCL, $201 \times 20$. (d) MUSCL, $801 \times 801$.
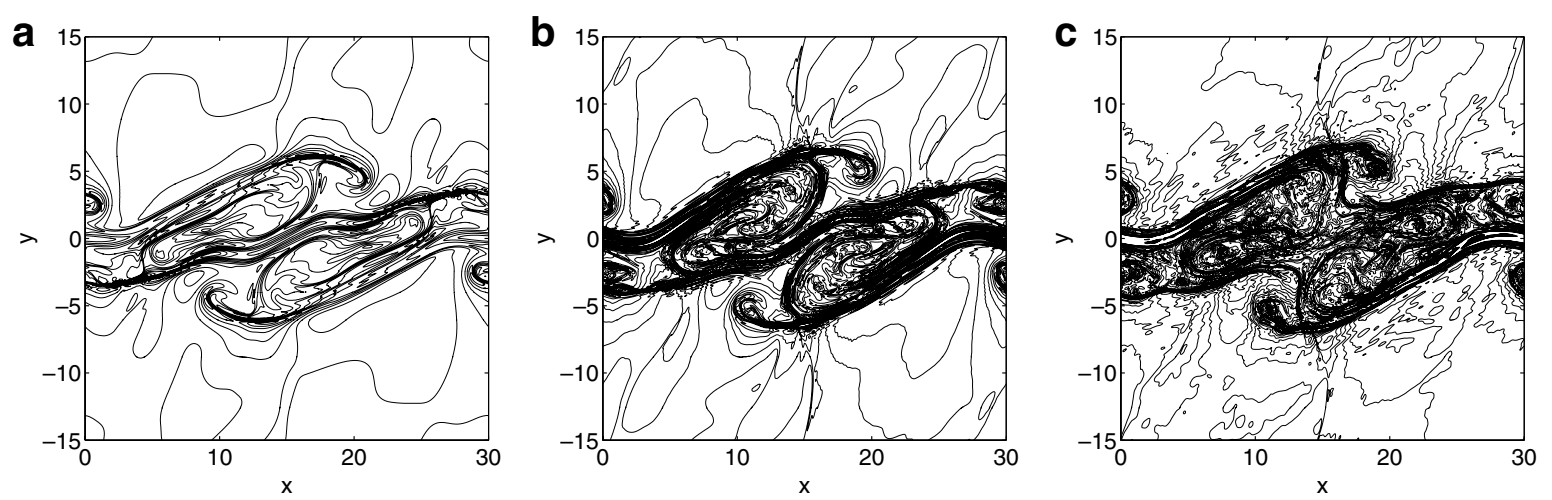

Fig. 12. Temperature contours of ACM66 for three $\beta_{h}$ for non-ideal MHD with $R e=10^{5}$ and conductivities $\sigma=10^{3}, 10^{5}$ using a $801 \times 801$ grid at $T=90$. (a) $\sigma=10^{3}, \beta_{h}=0.1, \operatorname{Re}=10^{5}$ (b) $\sigma=10^{5}, \beta_{h}=0.2, \operatorname{Re}=10^{5}$ (c) $\sigma=10^{5}, \beta_{h}=0.5, R e=10^{5}$.

plasma injection can alter the shock standoff distance and heating. The study also sheds some light on a simplified model related to solar wind physics. Both test cases indicate that the Hall term with large Hall coefficient poses a challenge in numerical modeling and simulation. Additional investigation is planned to overcome the numerical instability for large Hall coefficients. 


\section{Concluding remarks}

The efficiency and flexibility of the present class of low dissipative high order filter schemes are summarized with supporting numerical examples. The efficiency rests on the fact that even though the multistep filter can be applied after each stage of the Runge-Kutta method or after a full time step of the RungeKutta method, our numerical studies on many representative problems indicate that there is no difference in accuracy or stability among the two filter procedures. The latter procedure is very efficient. The major CPU time intensive part of the computation is the nonlinear filter. In fact, the latter filter procedure, in general, requires slightly more CPU time per time step (20\%) than the Harten-Yee and MUSCL schemes. This is due to the fact that all filter schemes WAV66-RK4 and ACM66-RK4 require only one Riemann solve per time step per direction (independent of the time discretizations of the base scheme step) as opposed to two Riemann solves per time step per direction by the MUSCL and Harten-Yee schemes using a second-order Runge-Kutta method. WENO5-RK4 requires at least twice the CPU time of all other methods since four Riemann solves per time step per direction are required by WENO5-RK4. RK4 stands for the classical fourth-order Runge-Kutta temporal discretization. Another gain in efficiency is that WAV66HYfi and WAV66wenofi exhibit similar accuracy for all of the considered test cases. In other words, the dissipative portion of second-order shock-capturing schemes as nonlinear filters is sufficient. Consequently, the complication of dealing with high order nonlinear filter with wider grid stencils and higher order numerical boundary treatment can be avoided.

Additional efficiency and accuracy improvement over standard high order shock-capturing schemes based on using the wavelet decomposition as a stand-alone module make the method and resulting software very flexible. One of the key advantages of the wavelet flow sensor filter method for problems with physical dissipation is that the more scales that are resolved, the less filter is utilized, thereby gaining accuracy and computation time. In the limit when all scales are resolved, we are left with a "pure" non-dissipative centered (or very low dissipative) high order spatial scheme. If instead the inviscid part of the equations had been discretized by a scheme with an advanced numerical dissipation model, e.g., the TVD, ENO and WENO schemes, the expensive computation of the numerical dissipation would have been made everywhere in the computational domain, even when dominated by physical dissipation.

From the numerical examples, the two filter methods WAV66 \& ACM66 employed the same nonlinear dissipation (e.g., the dissipative portion of TVD22, MUSCL or WENO5) and require similar CPU time, and yet the filter methods are far more accurate than the commonly used high-resolution shock-capturing methods. In most cases, in order to obtain a grid converged solution, standard second-order shock-capturing schemes and WENO5 required at least twice as many grid points in each spatial direction as ACM66 and WAV66. In three space dimensions, this means saving almost a factor 16 in execution time, since the grid can have 8 times fewer points, and the time step can be almost twice as large. Although no figure is shown, studies show that for a second-order base scheme filter method, improved accuracy was also realized in many multiscale shock/turbulence interactions. However, the improvement in accuracy is more pronounced if one uses the fourth-order or sixth-order base scheme which costs only slightly more CPU time (using the same second-order nonlinear filter). In fact, instead of nonlinear filtering, employing the flow sensor inside standard shock-capturing methods (for the dissipative portion) also results in improved accuracy (see [1]).

From our recent work, we believe that our numerical method and the accompanying research code have reached the proof of concept stage for a wide spectrum of multiscale shock/turbulence flows in generalized geometries. However, efficient and accurate numerical simulation of complex multiscale fluid and plasma flows containing strong shocks and high shear turbulence mixings remains computationally very challenging due to the wide range of temporal and spatial length scales. Capturing this type of interaction efficiently requires novel algorithms, stable treatment of stiffness due to the wide range of temporal and spatial scales, complex geometry handling, stable and accurate numerical treatment of physical and grid interface boundaries, local grid refinement, and effective use of software tools which allow the full benefit of the new algorithms to be realized on terascale and petascale supercomputer architectures. The recent development of our filter scheme is the first step to validate the advantage of the proposed schemes for multiscale gas dynamics/MHD problems over standard high-order shock-capturing methods. Future work includes implicit 
temporal treatment and multiblock/embedded grid capabilities for the simulation of practical gas dynamics and plasma physics.

\section{Acknowledgement}

The financial support from the NASA Fundamental Aeronautics Program for the first author is gratefully acknowledged.

\section{Appendix}

For our numerical experiments, the wavelet coefficient $w_{m, j}$ is computed numerically by a recursive procedure, which is a second-order B-spline wavelet or a modification of Harten's multi-resolution scheme [38] to be discussed shortly. We can express the algorithm as follows. Introduce the grid operators

$$
\begin{aligned}
& A f_{j}=\sum_{k=-p}^{q} d_{k} f_{j+k}, \\
& D f_{j}=\sum_{k=-p}^{q} c_{k} f_{j+k},
\end{aligned}
$$

and its $m$ th level expanded versions

$$
\begin{aligned}
& A_{m} f_{j}=\sum_{k=-p}^{q} d_{k} f_{j+2^{m} k}, \\
& D_{m} f_{j}=\sum_{k=-p}^{q} c_{k} f_{j+2^{m} k},
\end{aligned}
$$

where the integers $p$ and $q$ and the coefficients $d_{k}$ and $d_{k}$ are related to the chosen $\psi(x)$ and $\phi(x)$, and can be determined from them. Here, $\phi(x)$ is the so-called scaling function of the multiresolution wavelets.

The $m$ th level of wavelet coefficients can be written as

$$
w_{m, j}=\left\langle f, \psi_{m, j}\right\rangle=D_{m-1} A_{m-2} A_{m-3} \ldots A_{0} f_{j}, \quad m=1,2, \ldots
$$

Once the coefficients $d_{k}$ and $d_{k}$ are determined, the computation is a very standard application of grid operators. In practice, we only use $m_{0}=3$. To be able to compute up to the boundary, we use one sided versions of the given operators. This seems to work well in practice, although it is not covered by the wavelet framework described above.

\section{Detectors from the B-spline wavelet basis function}

Developing the best suited wavelets that can characterize all of the flow features might involve the switching or blending of more than one mother wavelet $\psi(x)$ and scaling function $\phi(x)$, especially if one needs to distinguish turbulent fluctuations from shock/shear and/or spurious high frequency oscillations. The mother wavelet function used in [13] and described in detail in [24] meets some of our requirements. It is obtained from second order B-splines.

$$
\psi(x)= \begin{cases}0 & x>1, \\ -2(x-1)^{2} & 1 / 2<x<1, \\ -4 x(1-x)+2 x^{2} & 0<x<1 / 2, \\ -4 x(1+x)-2 x^{2} & -1 / 2<x<0, \\ 2(x+1)^{2} & -1<x<-1 / 2, \\ 0 & x<-1 .\end{cases}
$$


For this wavelet (17), there exists a scaling function, given by

$$
\phi(x)= \begin{cases}0 & x>2, \\ \frac{1}{2}(x-2)^{2} & 1<x<2, \\ -(x-1 / 2)^{2}+3 / 4 & 0<x<1, \\ \frac{1}{2}(x+1)^{2} & -1<x<0, \\ 0 & x<-1 .\end{cases}
$$

The normalization is such that the integral of the scaling function above is equal to one. The functions above are standard, and can be found in [4]. The scaling function differs by a shift from the scaling function used in [13], but the important relations

$$
\begin{aligned}
\phi(x) & =\frac{1}{4} \phi(2 x+1)+\frac{3}{4} \phi(2 x)+\frac{3}{4} \phi(2 x-1)+\frac{1}{4} \phi(2 x-2), \\
\psi(x) & =\phi(2 x+1)-\phi(2 x),
\end{aligned}
$$

hold, and give the grid operators

$$
\begin{aligned}
& A f_{j}=\left(f_{j-1}+3 f_{j}+3 f_{j+1}+f_{j+2}\right) / 8, \quad j=2, \ldots, N-2, \\
& D f_{j}=\left(f_{j-1}-f_{j}\right) / 2 \quad j=2, \ldots, N .
\end{aligned}
$$

Note that this wavelet stencil is not symmetric. In general, the wavelet coefficients involve points from $p 2^{m_{0}-1}$ to $-q 2^{m_{0}-1}$, giving a stencil of totally $(p+q) 2^{m_{0}-1}+1$ points.

\section{Detectors from the redundant form of Harten multiresolution wavelet}

For the redundant form of Harten multiresolution wavelet there is more than one choice for the interpolation function. See Sjögreen [37] for a discussion. The exact form of the method for the computations in this article is

$$
\begin{aligned}
& A f_{j}=\left(f_{j-1}+f_{j+1}\right) / 2, \quad j=2, \ldots, N-1, \\
& D f_{j}=f_{j}-A f_{j}, \quad j=2, \ldots, N-1 .
\end{aligned}
$$

The above choice was made in order to have a simple and efficient method. The stencil is narrower than for the B-spline formulas that were given previously. With the formula above we also get a symmetric stencil, which is more natural if the other parts of the computation, such as difference approximations of PDEs are done by symmetric formulas. Furthermore, symmetry makes periodic BCs somewhat easier to implement. Note that the absence of symmetry for either the scaling function or the wavelet can lead to phase distortion. This can be shown to be important in signal processing applications.

\section{Multi-dimensional wavelets}

The computation of multi-dimensional wavelets is quite expensive, especially in 3D. A simple minded efficient way is to evaluate the wavelet coefficients dimension-by-dimension. This means that we get two set of wavelet coefficients $w_{m, j}^{x}(y)$ and $w_{m, k}^{y}(x)$, where now $(j, k)$ is the position and $m$ is the scale. The precise definition is

$$
\begin{aligned}
w_{m, j}^{x}(y) & =\int f(x, y) \psi_{m, j}(x) \mathrm{d} x, \\
w_{m, k}^{y}(x) & =\int f(x, y) \psi_{m, k}(y) \mathrm{d} y .
\end{aligned}
$$

Thus, the dimension-by-dimension approach involved only terms evaluated as finite differences in the $x$-direction and terms which are evaluated in the $y$-direction. We then use the $w_{m, j}^{x}(y)$ coefficients for the $x$-direction computation, and the $y$-coefficients for the $y$-direction computation. 


\section{Shock/shear wavelet sensor}

For the numerical experiments presented in the next section the wavelet sensor is obtained by computing a vector of the approximated Lipschitz exponent of a chosen vector function to be sensed with a suitable multiresolution non-orthogonal wavelet basis function. Here, "vectors or variables to be sensed" means the represented vectors or variables that are suitable for the extraction of the desired flow physics. The variables to be sensed can be the density, the combination of density and pressure, the characteristic variables, the jumps in the characteristic variables $\tilde{\alpha}_{j+1 / 2}^{l}$, or the entropy variable vector $W([13,54])$.

For example, if the characteristic variables are the chosen vector to be sensed by the wavelet approach, the flow sensor $\mathscr{S}_{j+1 / 2}^{l}$ can be defined as

$$
\mathscr{S}_{j+1 / 2}^{l}=\tau\left(\alpha_{j+1 / 2}^{l}\right) \text {, }
$$

where $\alpha_{j+1 / 2}^{l}$ is the estimated Lipschitz exponent of the $l$ th characteristic component with $l=1,2,3,4$, the four characteristic waves. $\tau(\alpha)$ is a sensing function which decreases from $\tau(0)=1$ to $\tau(1)=0$ (for the aforementioned type of wavelets). Noted that the $k$ index is omitted (for the $2 \mathrm{D}$ case) for simplicity.

If we instead base the exponent estimate on point centered quantities, we will use the sensor function

$$
\mathscr{S}_{j+1 / 2}^{l}=\max \left(\tau\left(\alpha_{j}^{l}\right), \tau\left(\alpha_{j+1}^{l}\right)\right) \text {. }
$$

If the exponent estimate is based on other quantities than the characteristic variables, (e.g., density and pressure), we use the switch

$$
\mathscr{S}_{j+1 / 2}=\max _{l} \mathscr{S}_{j+1 / 2}^{l},
$$

where the maximum is taken over all components of the waves used in the estimate. In this case, the switch is the same for all characteristic fields. The function $\tau(\alpha)$ should be such that $\tau(0)=1$, and $\tau(1)=0$. Three options considered are

$$
\begin{aligned}
\tau(\alpha) & = \begin{cases}1 & \alpha<\alpha_{0} \\
0 & \alpha \geqslant \alpha_{0}\end{cases} \\
\tau(\alpha) & =\frac{1}{2}+\frac{1}{\pi} \arctan K\left(\alpha_{0}-\alpha\right) \\
\tau(\alpha) & =\max \left\{0, \min \left[1,(\alpha-1) /\left(\alpha_{0}-1\right)\right]\right\} .
\end{aligned}
$$

Here, $\alpha_{0}$ is a cutoff exponent to be chosen. For the arctan function the values 0 and 1 are not attained, but we take the constant $K$ large enough so that the function is close to zero for $\alpha>1$, and close to one for $\alpha<0$. We have tried values for $K$ in the interval [200,500]. Alternatively, one can integrate the actual $\alpha$ value into the sensor function instead of using the same amount of numerical dissipation at the cutoff exponent.

After some experimentation we have found that switching on the dissipation at the grid points where $\alpha<0.5$ works well, i.e.,

$$
\tau(\alpha)= \begin{cases}1 & \alpha<0.5 \\ 0 & \alpha \geqslant 0.5\end{cases}
$$

In fact the method does not seem to be very sensitive to the exact value of cutoff $\alpha_{0}$, (for $0.4 \leqslant \alpha_{0} \leqslant 0.6$ ) for all the test cases considered. Furthermore, the same cut off value, 0.5 , works well for all problems we have tried. Experiments with smoothed step functions do not give very different results.

\section{References}

[1] M.F. Barone, C.J. Roy, Evaluation of detached eddy simulation for turbulent wake applications, in: AIAA Paper 2005-0504, 43rd Aerospace Science Meeting and Exhibit, Reno, NV, 2005.

[2] M. Brio, C.C. Wu, An upwind differencing scheme for the equations of ideal magnetohydrodynamics, J. Comput. Phys. 75 (1988) 400-422.

[3] W. Dai, P.R. Woodward, A simple finite difference scheme for multidimensional magnetohydrodynamical equations, J. Comput. Phys. 142 (1998) 331-369. 
[4] I. Daubechies, Ten Lectures on Wavelets, CBMS-NSF Regional Conference Series in Applied Mathematics, SIAM, 1992 , No 61.

[6] W.S. Don, C.B. Quillen, Numerical simulation of shock-cylinder interactions, J. Comput. Phys. 122 (1995) $244-265$.

[7] W.S. Don, D. Gottlieb, Spectral simulation of supersonic reactive flows, SIAM J. Numer. Anal. 35 (1998) $2370-2384$.

[8] C.R. Evans, J.F. Hawley, Simulation of magnetohydrodynamic flows: a constrained transport method, Astrophys. J. 332 (1988) 659677.

[9] M. Farge, Wavelet transforms and their applications to turbulence, Ann. Rev. Fluid Mech. 24 (1992) 395.

[10] D.V. Gaitonde, M.R. Visbal, Further development of a Navier-Stokes solution procedure based on higher-order formulas, AIAA Paper 99-0557, 1999.

[11] D.V. Gaitonde, Development of a solver for 3D non-ideal magnetogasdynamics, AIAA Paper 99-3610, 1999.

[12] G. Gallice, Systéme D’Euler-Poisson, Magnétohydrodynamique et Schemas de Roe, PhD Thesis, L’Université Bordeaux I, 1997.

[13] M. Gerritsen, P. Olsson, Designing an efficient solution strategy for fluid flows I, J. Comput. Phys. 129 (1996) $245-262$.

[14] S.K. Godunovm, Symmetric form of the equations of magnetohydrodynamics, Numer. Meth. Mech. Continuum Medium 13 (1) (1972) 26-34.

[15] A. Harten, Multiresolution algorithms for the numerical solution of hyperbolic conservation laws, Comm. Pure Appl. Math. 48 (1995) 1305-1342.

[16] A. Harten, J. Hyman, Self-adjusting grid methods for one-dimensional hyperbolic conservation laws, J. Comput. Phys. 50 (1983) 235.

[17] G.-S. Jiang, C.-W. Shu, Efficient implementation of weighted ENO schemes, J. Comput. Phys 126 (1996) 202.

[19] S.G. Mallat, A Wavelet Tour of Signal Processing, second ed., Academic Press, San Diego, 1999.

[20] S.G. Mallat, Multifrequency channel decompositions of images and wavelet models, IEEE Trans. Acoust. Speech Signal Process. 37 (1989) 2091.

[21] S.G. Mallat, Multiresolution approximations and wavelet orthonormal bases of $L^{2}(R)$, Trans. Am. Math. Soc. 315 (1989) 69.

[22] S.G. Mallat, A theory for multiresolution signal decomposition: the wavelet representation, IEEE Trans. Patt. Anal. Mach. Intell. 11 (1989) 674.

[23] S.G. Mallat, W.L. Hwang, Singularity detection and processing with wavelets, IEEE Trans. Inform. Theory 38 (1992) 617.

[24] S.G. Mallat, S. Zhong, Characterization of signals from multiscale edges, IEEE Trans. Pattern Anal. Mach. Intell. 14 (1992) $710-732$.

[25] McBride, B.J., Heimel, S., Ehlers, J.G., Gorden, S., Thermodynamics properties to $6000{ }^{\circ} \mathrm{K}$ for 210 substances involving the first 18 elements, NASA SP-3002, 1963.

[26] Y.Y. Meshkov, Instability of a shock wave accelerated interface between two gases, NASA Tech. Trans. NASA TT F-13074, 1970.

[27] R. Samtaney, Suppression of the Richtmyer-Meshkov instability in the presence of a magnetic field, Phys. Fluids 15 (2003) L53-L56.

[28] H. Nessyahu, E. Tadmor, Non-oscillatory central differencing for hyperbolic conservation laws, J. Comput. Phys. 87 (1990) $408-463$.

[29] J. Nordstrom, M.H. Carpenter, Boundary and interface conditions for high-order finite-difference schemes applied to the Euler and Navier-Stokes equations, J. Comput. Phys. 148 (1999) 621-645.

[30] P. Olsson, Summation by parts, projections and stability I, Math. Comput. 64 (1995) 1035-1065.

[31] V. Perrier, T. Philipovitch, C. Basdevant, Wavelet Spectra Compared to Fourier Spectra, Publication of ENS, Paris, 1999.

[32] K.G. Powell, An approximate riemann solver for magnetohydrodynamics (that works in more than one dimension), ICASE-Report 94-24, NASA Langley Research Center, April 1994.

[33] J. Qiu, B.C. Khoo, C.-W. Shu, A numerical study for the performance of the Runge-Kutta discontinuous Galerkin method based on different numerical fluxes, J. Comput. Phys. 212 (2006) 540-565.

[34] R.D. Richtmyer, Taylor instability in shock acceleration of compressible fluids, Commun. Pure Appl. Math. 13 (1960) 297.

[35] N.D. Sandham, Q. Li, H.C. Yee, Entropy splitting for high-order numerical simulation of compressible turbulence, J. Comput. Phys. 178 (2002) 307-322.

[36] B. Sjögreen, High order centered difference methods for the compressible Navier-Stokes equations, J. Comput. Phys. 117 (1995) 6778.

[37] B. Sjögreen, Numerical experiments with the multiresolution scheme for the compressible Euler equations, J. Comput. Phys. 117 (1995) 251.

[38] B. Sjögreen, H.C. Yee, Multiresolution wavelet based adaptive numerical dissipation control for shock-turbulence computation, RIACS Technical Report TR01.01, NASA Ames research center (Oct 2000); J. Sci. Comput. 20 (2004) 211-255.

[40] B. Sjögreen, H.C. Yee, Efficient low dissipative high order schemes for multiscale MHD flows, I: Basic theory, AIAA 2003-4118, in: Proceedings of the 16th AIAA/CFD Conference, June 23-26, Orlando, FL, 2003.

[41] B. Sjögreen, H.C. Yee, Grid convergence of high order methods for multiscale complex unsteady viscous compressible flows, J. Comput. Phys. 185 (2003) 1-26.

[42] B. Sjögreen, H.C. Yee, Low dissipative high-order numerical simulations of supersonic reactive flows, Int. J. Numer. Meth. Fluids 43 (2003) 1221-1238.

[43] B. Sjögreen, H.C. Yee, A numerical study of resistivity and hall effects for a compressible MHD model, AIAA Paper No. 5046-2005, in: 36th AIAA Plasmadynamics and Lasers Conference, June 6-9, Toronto, Ont., Canada, 2005.

[44] B. Sjögreen, H.C. Yee, Performance of high order filter methods for a Richtmyer-Meshkov Instability, in: Proceedings of the ICCFD4 Conference, July 10-14, Gent, Belgium, 2006.

[46] G. Strang, Wavelet transforms versus fourier transforms, Bull. Am. Math. Soc. (N.S.) 28 (1993) 288.

[47] G. Strang, Wavelet and dilation equations: a brief introduction, SIAM Rev. 31 (1989) 614.

[48] R.A. Svehla, Estimated viscosities and thermal conductivities of gases at high temperatures, NASA Report TR R-132, 1962.

[49] M. Vinokur, H.C. Yee, Extension of efficient low dissipative high order schemes for 3D curvilinear moving grids, NASA TM 209598, June 2000; in: D.A. Caughey, M. Hafez (Eds.), Frontiers of Computational Fluid Dynamics, World Scientific, 2002 , pp. 129-164. 
[50] V. Wheatley, D.I. Pullin, R. Samtaney, Regular shock refraction at an oblique planar density interface in magnetohydrodynamics, J. Fluid Mech. 522 (2005) 179-214.

[51] C.R. Wilke, A viscosity equation for gas mixtures, Chem. Phys. 18 (1950) 517-519.

[52] H.C. Yee, A Class of High-Resolution Explicit and Implicit Shock-Capturing Methods, VKI Lecture Series 1989-04, March 6-10, 1989, also NASA TM-101088, Feb. 1989.

[53] H.C. Yee, N.D. Sandham, M.J. Djomehri, Low dissipative high order shock-capturing methods using characteristic-based filters, J. Comput. Phys. 150 (1999) 199-238.

[54] H.C. Yee, M. Vinokur, M.J. Djomehri, Entropy splitting and numerical dissipation, J. Comput. Phys. 162 (2000) 33-81.

[55] H.C.Yee, B. Sjögreen, Designing adaptive low dissipative high order schemes for long-time integrations for long-time integrations, in: D. Drikakis, B. Geurts (Eds.), Turbulent Flow Computation, Kluwer Academic Publisher (2002); also RIACS Technical Report TR01-28, December 2001.

[56] H.C. Yee, B. Sjögreen, Divergence free high order filter methods for the compressible MHD equations, in: Proceedings of the International Conference on High Performance Scientific Computing, March 10-14, Hanoi, Vietnam, 2003.

[57] H.C. Yee, B. Sjögreen, Efficient low dissipative high order scheme for multiscale MHD flows, II: Minimization of Div(B) numerical error, RIACS Technical Report TR03.10, July, 2003, NASA Ames Research Center; also, J. Sci. Comput. (2005), DOI: 10.1007/ s10915-005-9004-5.

[58] H.C. Yee, B. Sjögreen, Adaptive numerical dissipation control in high order schemes for 3D non-ideal MHD, in: Proceedings of the ICCFD3, July 12-16, Toronto, Canada, 2004.

[59] H.C. Yee, B. Sjögreen, Nonlinear filtering and limiting in high order methods for ideal and non-ideal MHD, in: Proceedings of the ICOSAHOM, July 21-25, Brown University, RI; J. Scient. Comput. 27 (2006) 507-521.

[60] H.C. Yee, B. Sjögreen, Performance of adaptive numerical dissipation control in high order methods for the resistive MHD equations, in: Proceedings of the HYP2004, September 13-17, Osaka, Japan, 2004.

[61] H.C. Yee, B. Sjögreen, Nonlinear filtering in compact high order schemes, in: Proceedings of the 19th ICNSP and 7th APPTC Conference, Nara, Japan, July 11-15, 2005. Extended version entitled "Adaptive Filtering and Limiting in Compact High Order Methods for Multiscale Gas Dynamics and MHD Systems," Invited paper for a special issue of Computers and Fluids in Honor of Prof. M. Hafex's 60th-Birthday.

[63] H.C. Yee, B. Sjögreen, Simulation of Richtmyer-Meshkov instability by sixth-order filter methods, in: Proceedings of the 17th International Shock Interaction Symposium, University of Rome, Rome, Italy, 2006, also, Shock Waves Journal, submitted for publication. 